\title{
The interaction of young massive stars with their environment ${ }^{\star} \star \star$
}

\section{A millimeter and submillimeter line study of NGC 6334 FIR II}

\author{
J. S. Zhang ${ }^{1,2,3}$, C. Henkel $^{1}$, R. Mauersberger ${ }^{4}$, Y.-N. Chin ${ }^{5}$, K. M. Menten ${ }^{1}$, A. R. Tieftrunk ${ }^{6}$, and A. Belloche ${ }^{1}$ \\ 1 Max-Planck-Institut für Radioastronomie, Auf dem Hügel 69, 53121 Bonn, Germany \\ e-mail: dennyzhang998@hotmail.com \\ 2 Purple Mountain Observatory, Chinese Academy of Sciences, NanJing 210008, PR China \\ 3 Center for Astrophysics, GuangZhou University, GuangZhou 510006, PR China \\ 4 Instituto de Radioastronomía Millimétrica (IRAM), Avenida Divina Pastora 7, Local 20, 18012 Granada, Spain \\ 5 Department of Physics, Tamkang University, 251-37 Tamsui, Taipeh County, Taiwan \\ ${ }^{6}$ Im Acker 21b, 56072 Koblenz, Germany
}

Received 29 June 2006 / Accepted 7 January 2007

\section{ABSTRACT}

\begin{abstract}
Using the 15-m Swedish ESO Sub-millimeter Telescope (SEST), CO, HCN, and $\mathrm{HCO}^{+}$observations of the galactic star-forming region NGC 6334 FIR II are presented, complemented by [C I ${ }^{3} \mathrm{P}_{1}-{ }^{3} \mathrm{P}_{0}$ and ${ }^{3} \mathrm{P}_{2}-{ }^{3} \mathrm{P}_{1}$ data from the Atacama Pathfinder Experiment (APEX 12-m telescope). Embedded in the extended molecular cloud and associated with the HII region NGC 6334-D, there is a molecular "void". [C I] correlates well with ${ }^{13} \mathrm{CO}$ and other molecular lines and shows no rim brightening relative to molecular cloud regions farther off the void. While an interpretation in terms of a highly clumped cloud morphology is possible, with photon dominated regions (PDRs) reaching deep into the cloud, the data do not provide any direct evidence for a close association of [CI] with PDRs. Kinetic temperatures are $\sim 40-50 \mathrm{~K}$ in the molecular cloud and $\gtrsim 200 \mathrm{~K}$ toward the void. $\mathrm{CO}$ and $[\mathrm{CI}]$ excitation temperatures are similar. A comparison of molecular and atomic fine structure line emission with the far infrared and radio continuum as well as the distribution of $2.2 \mu \mathrm{m} \mathrm{H}_{2}$ emission indicates that the well-evolved $\mathrm{H}$ II region expands into a medium that is homogeneous on pcscales. If the $\mathrm{H}_{2}$ emission is predominantly shock excited, both the expanding ionization front (classified as subsonic, "D-type") and the associated shock front farther out (traced by $\mathrm{H}_{2}$ ) can be identified, observationally confirming for the first time a classical scenario that is predicted by evolutionary models of HII regions. Integrated line intensity ratios of the observed molecules are determined, implying a mean $\mathrm{C}^{18} \mathrm{O} / \mathrm{C}^{17} \mathrm{O}$ abundance ratio of $4.13 \pm 0.13$ that reflects the ${ }^{18} \mathrm{O} /{ }^{17} \mathrm{O}$ isotope ratio. This ratio is consistent with values determined in nearby clouds. Right at the edge of the void, however, the oxygen isotope ratio might be smaller.
\end{abstract}

Key words. ISM: abundances - ISM: atoms - ISM: H II regions - ISM: individual objects: NGC 6334 FIR II - ISM: molecules radio lines: ISM

\section{Introduction}

Young massive stars greatly affect their environment, either impeding or triggering future star formation. Theory (e.g., Franco et al. 1990) predicts that soon after the radiation field of a hot massive star has been turned on, its energetic photons create a supersonic ("R-type") ionization front that moves through the surrounding molecular gas, leaving it hot and ionized. The speed of this front is continuously reduced by geometric dilution and recombination. At a time when the speed is not much larger than the speed of sound, i.e., when the "initial" Strömgen radius is reached, the large pressure gradient across the ionization front is supposed to drive a supersonic shock wave that sweeps, accelerates, and compresses the surrounding medium. The ionization front further decelerates, becomes subsonic ("D-type"), and follows the shock front, while neutral gas accumulates in the interphase between the fronts.

\footnotetext{
* Based on observations with the Atacama Pathfinder EXperiment telescope (APEX, Chile) and the Swedish-ESO Sub-millimeter Telescope (SEST, ESO/La Silla, Chile).

$\star \star$ Figure A.1 is only available in electronic form at http://www . aanda.org
}

Complications arise when the surrounding gas is clumpy. If the ionization or shock front encounters a strong negative density gradient, e.g., at the edge of the parent cloud, the ionized gas may expand supersonically ("champagne phase"). Another complication arises when young massive stars form in clusters so that scenarios based on a single dominating star may be misleading. Poorly collimated mainly low velocity stellar winds from young massive stars may also contribute significantly to the budget of incoming energy (e.g., Tenorio-Tagle 1988; Churchwell 2002).

Since it is known that molecular gas is highly clumped (e.g., Wilson \& Walmsley 1989), the less energetic UV radiation $(\sim 6-13.6 \mathrm{eV})$ emitted by the young star(s) may leak deeply into the cloud. Such "photon-dominated regions" (PDRs) may thus make up a large fraction of the volume of a star-forming cloud (e.g., Hollenbach \& Tielens 1997). The UV radiation from the star as well as associated shock fronts heat the dust and the gas. Molecules will be photodissociated and surface layers of dust grains will evaporate. The particles generated in such ways are ready to form new molecular species characteristic of a PDR or shocked environment.

Many star-forming regions have been studied in some detail. Usually it is found that the geometry of the scrutinized region is irregular. In the following we present a study of an object of 
particularly simple geometry, the periphery of an almost circular region devoid of molecular gas. This region forms the eastern part of NGC 6334 FIR II, the outstanding source of far infrared emission in the prominent southern star forming region NGC 6334 (Loughran et al. 1986). Here we present maps of the region in a number of molecular transitions $(\lambda \sim 3$ and $1.3 \mathrm{~mm}$ ) that are complemented by profiles from the 492 and $809 \mathrm{GHz}$ lines of neutral carbon $(\mathrm{CI})$. Observational methods are described in Sect.2. New data are presented in Sect. 3, while Sect. 4 provides physical parameters, including isotope ratios determined from CO. Section 5 summarizes the main results.

\section{Observations}

\subsection{SEST observations}

The $J=1-0$ lines of ${ }^{12} \mathrm{CO},{ }^{13} \mathrm{CO}, \mathrm{C}^{18} \mathrm{O}, \mathrm{C}^{17} \mathrm{O}, \mathrm{HCN}$, and $\mathrm{HCO}^{+}$, as well as the $J=2-1$ line of ${ }^{13} \mathrm{CO}$ have been observed with the 15-m Swedish-ESO Sub-millimeter Telescope (SEST, Booth et al. 1989) in May $1993\left(\mathrm{C}^{18} \mathrm{O}\right)$ and May 1994. For the $3 \mathrm{~mm}$ observations, a Schottky receiver was used. System temperatures, $T_{\text {sys }}$, were typically $480 \mathrm{~K}$ on a main-beam brightness temperature $\left(T_{\mathrm{mb}}\right)$ scale. $1.3 \mathrm{~mm}$ data were obtained with an SIS receiver with $T_{\text {sys }} \sim 1600 \mathrm{~K}$.

The chopper wheel method was used for calibration. The intensity scale was converted to a $T_{\mathrm{mb}}$ scale (see Downes 1989; Rohlfs \& Wilson 1996) assuming main-beam efficiencies of $0.75,0.70$, and 0.60 at $v \sim 90 \mathrm{GHz}, 110-115 \mathrm{GHz}$, and $220 \mathrm{GHz}$, respectively (SEST Handbook, 1993). The full width to half power (FWHP) beam-width was 57" for $\mathrm{HCN}$ and $\mathrm{HCO}^{+}, 45^{\prime \prime}$ for the $J=1-0$ lines of $\mathrm{CO}$ and its rare isotopomers, and $24^{\prime \prime}$ for the $J=2-1$ transition of ${ }^{13} \mathrm{CO}$.

The pointing of the telescope was regularly determined by mapping the $\mathrm{SiO}$ masers toward VX Sgr and AH Sco. We estimate the pointing to be correct within $10^{\prime \prime}$ (but see $\mathrm{C}^{17} \mathrm{O}$ in Sect.4.7), while the calibration uncertainty is estimated to be $\pm 15 \%$. As backends, we employed acousto-optical spectrometers with 1000 or 1652 contiguous channels, a channel spacing of $43 \mathrm{kHz}$, and a spectral resolution of $80 \mathrm{kHz}(0.27$, 0.21 , and $0.11 \mathrm{~km} \mathrm{~s}^{-1}$ at 90,112 , and $220 \mathrm{GHz}$, respectively). The $J=1-0$ transitions of ${ }^{12} \mathrm{CO}$ and ${ }^{13} \mathrm{CO}$ were measured in a dual frequency switching mode, with frequency offsets of $\pm 15 \mathrm{MHz}$. The other measurements were carried out in a position switching mode, the reference position being at an offset of $(\Delta \alpha, \Delta \delta)=\left(-20^{\prime}, 20^{\prime}\right)$. The integration times per point were usually 15 to $60 \mathrm{~s}$; only for $\mathrm{C}^{17} \mathrm{O}$ were integration times longer, with typically 5 min per position. From all the spectra, linear baselines have been subtracted. A summary of observational parameters is presented in Table 1. Spectra reduced using the GILDAS software (e.g., Guilloteau \& Lucas 2000) are shown in Fig. A. $1^{1}$ of the Appendix that is available electronically.

\subsection{APEX observations}

The ${ }^{3} \mathrm{P}_{1}-{ }^{3} \mathrm{P}_{0}$ and ${ }^{3} \mathrm{P}_{2}-{ }^{3} \mathrm{P}_{1}$ fine-structure lines of neutral atomic carbon (C I) were observed in April 2006, using FLASH (First Light Apex Submillimeter Heterodyne instrument; Heyminck et al. 2006) on the 12-m APEX (Atacama Pathfinder Experiment; Güsten et al. 2006) telescope. FLASH is a dualchannel heterodyne instrument operating simultaneously in the

1 Throughout the paper, B1950.0 coordinates were used to facilitate comparisons with previously published results (e.g., Brooks \& Whiteoak 2001; Ezoe et al. 2006; and earlier publications).
Table 1. Observed lines toward NGC 6334.

\begin{tabular}{lrrrrr}
\hline \hline Transition & $\begin{array}{c}\text { Frequency } \\
(\mathrm{GHz})\end{array}$ & $\begin{array}{c}\text { Beam } \\
\left({ }^{\prime \prime}\right)\end{array}$ & $\begin{array}{r}L^{a} \\
(\mathrm{pc})\end{array}$ & $\eta_{\mathrm{mb}}^{b}$ & \multicolumn{1}{c}{$\begin{array}{c}\text { Time }^{c} \\
(\mathrm{~s})\end{array}$} \\
\hline SEST 15-m & & & & & \\
$\mathrm{HCN}(1-0)$ & 88.632 & 57 & 0.48 & 0.75 & 30 \\
$\mathrm{HCO}^{+}(1-0)$ & 89.188 & 57 & 0.48 & 0.75 & 30 \\
${ }^{12} \mathrm{C}^{18} \mathrm{O}(1-0)$ & 109.782 & 45 & 0.38 & 0.7 & 60 \\
${ }^{13} \mathrm{C}^{16} \mathrm{O}(1-0)$ & 110.201 & 45 & 0.38 & 0.7 & 15 \\
${ }^{12} \mathrm{C}^{17} \mathrm{O}(1-0)$ & 112.359 & 45 & 0.38 & 0.7 & 300 \\
${ }^{12} \mathrm{C}^{16} \mathrm{O}(1-0)$ & 115.271 & 45 & 0.38 & 0.7 & 15 \\
${ }^{13} \mathrm{C}^{16} \mathrm{O}(2-1)$ & 220.399 & 24 & 0.20 & 0.6 & 30 \\
\hline${ }_{\mathrm{APEX} 12-\mathrm{P}}$ & & & & & \\
{$[\mathrm{C} \mathrm{I}]^{3} \mathrm{P}_{1}-{ }^{3} \mathrm{P}_{0}$} & 492.160 & 14 & 0.11 & 0.60 & 30 \\
{$[\mathrm{C} \mathrm{I}]^{3} \mathrm{P}_{2}-{ }^{3} \mathrm{P}_{1}$} & 809.341 & 8 & 0.07 & 0.43 & 30 \\
\hline
\end{tabular}

${ }^{a}$ To estimate the linear size of the respective beam, $D=1.7 \mathrm{kpc}$ is adopted (Neckel 1978).

${ }^{b}$ Main beam efficiencies; for references see Sect. 2.

${ }^{c}$ On-source integration times: For [C I], integration times are typically $30 \mathrm{~s}$ per scan, but the central position has been observed several times (see Sect. 2.2).

$420-500 \mathrm{GHz}$ and the $780-880 \mathrm{GHz}$ atmospheric windows. Double sideband system temperatures were $\sim 1000-2000 \mathrm{~K}$ and $3800-8000 \mathrm{~K}$ on a $T_{\mathrm{mb}}$ scale, respectively, mainly depending on elevation. The measurements were carried out in a position switching mode with the reference position at an offset of $(\Delta \alpha, \Delta \delta)=\left(20^{\prime}, 0^{\prime}\right)$. On-source integration times were 30 to 90 s per position; the central position was observed repeatedly to check calibration, resulting in a total on-source integration time of about $300 \mathrm{~s}$. Observational parameters are summarized in Table 1.

The data were calibrated to a $T_{\mathrm{mb}}$ scale adopting main-beam efficiencies of 0.60 and 0.43 and forward hemisphere efficiencies of 0.95 at $v \sim 492 \mathrm{GHz}$ and $809 \mathrm{GHz}$, respectively. Full width to half power (FWHP) beam-widths are 14" and $8^{\prime \prime}$ (Güsten et al. 2006). The absolute calibration uncertainty is estimated to be $\pm 25 \%$; the relative calibration uncertainty is about $\pm 15 \%$.

As backend a fast-fourier-transform spectrometer was used, containing 16384 channels and covering a bandwidth of $1 \mathrm{GHz}$ (Klein et al. 2006). The resulting channel spacing is $61 \mathrm{kHz}(0.04$ and $0.02 \mathrm{~km} \mathrm{~s}^{-1}$ at the lower and higher frequencies, respectively). Pointing and focus sources were SgrB2(N) and Venus.

\section{Results}

\subsection{Molecular line emission}

We mapped the large-scale distribution of the ${ }^{13} \mathrm{CO} J=$ $1-0$ emission over a region of roughly $10^{\prime} \times 30^{\prime}$ (Fig. 1a) covering almost the entire "molecular ridge" of NGC 6334 that is characterized by a position angle of about $45^{\circ}$ (e.g., Kraemer \& Jackson 1999). At a distance of $1.7( \pm 0.3) \mathrm{kpc}$ (Neckel 1978), $1^{\prime}$ corresponds to a linear size of $0.5 \mathrm{pc}$. The map spacing is $40^{\prime \prime}$ near the ridge and $2^{\prime}$ further away from the ridge. The northeastern part of the map shows a small inconspicious "void" surrounded by extended molecular emission. Covering this area that is marked by a square in Fig. 1a, maps of ${ }^{12} \mathrm{CO},{ }^{13} \mathrm{CO}, \mathrm{C}^{18} \mathrm{O}$, $\mathrm{C}^{17} \mathrm{O}, \mathrm{HCN}$, and $\mathrm{HCO}^{+}$were taken on a finer grid consisting of $13 \times 13$ spectra. These are shown in Figs. $1 \mathrm{~b}-\mathrm{h}$. The spacing of $20^{\prime \prime}$ implies full sampling for all lines except ${ }^{13} \mathrm{CO} J=2-1$. In these more detailed maps, the void becomes a prominent 


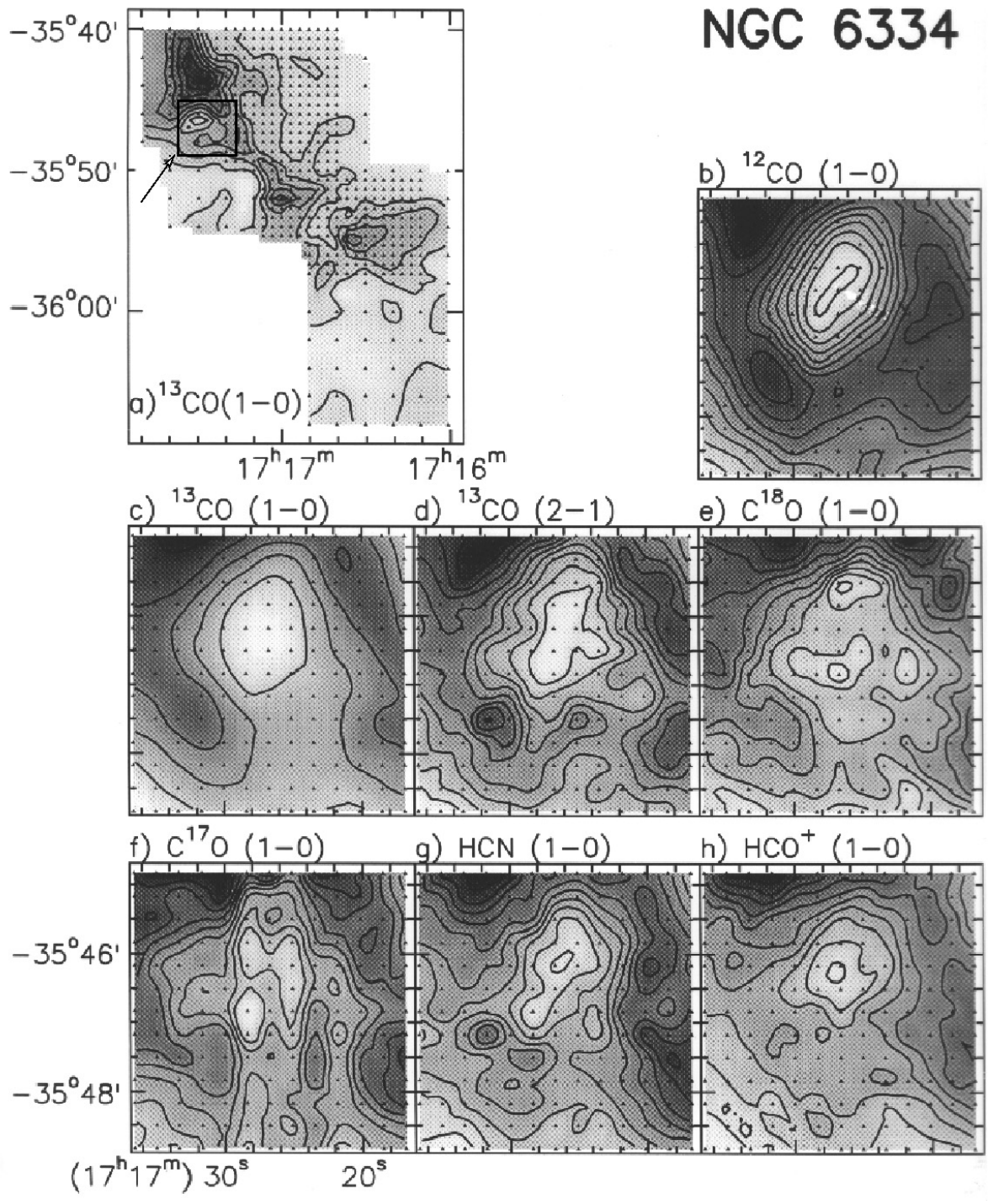

Fig. 1. Contour maps of integrated intensity, $\int T_{\mathrm{MB}} \mathrm{d} v$. $1^{\prime}$ corresponds to $0.5 \mathrm{pc}$. The measured positions are indicated by markers. a) Large-scale ${ }^{13} \mathrm{CO} J=1-0$ map. The lowest contour is $8 \mathrm{~K} \mathrm{~km} \mathrm{~s}^{-1}$, the contour increment is $18 \mathrm{~K} \mathrm{~km} \mathrm{~s}^{-1}$, and the rms noise is $1.11 \mathrm{~K} \mathrm{~km} \mathrm{~s}^{-1}$. The arrow points toward the area shown in the other maps of this figure, including the emission of seven molecular lines centered at $\alpha_{1950}=17^{\mathrm{h}} 17^{\mathrm{m}} 26^{\mathrm{s}} .7$, $\delta_{1950}=-35^{\circ} 46^{\prime} 50^{\prime \prime}\left(\alpha_{2000}=17^{\mathrm{h}} 20^{\mathrm{m}} 47^{\mathrm{s}} .9\right.$, $\left.\delta_{1950}=-35^{\circ} 49^{\prime} 48^{\prime \prime}\right)$. b) ${ }^{12} \mathrm{CO} J=1-0$ (lowest contour: $99 \mathrm{~K} \mathrm{~km} \mathrm{~s}^{-1}$, increment: $33 \mathrm{~K} \mathrm{~km} \mathrm{~s}^{-1}$, rms noise: $5.85 \mathrm{~K} \mathrm{~km} \mathrm{~s}^{-1}$ ). c) ${ }^{13} \mathrm{CO} J=1-0$ (lowest contour: $8 \mathrm{~K} \mathrm{~km} \mathrm{~s}^{-1}$, increment: $18 \mathrm{~K} \mathrm{~km} \mathrm{~s}^{-1}$, $\mathrm{rms}$ noise: $1.82 \mathrm{~K} \mathrm{~km} \mathrm{~s}^{-1}$ ). d) ${ }^{13} \mathrm{CO} J=2-1$ (lowest contour: $35 \mathrm{~K} \mathrm{~km} \mathrm{~s}^{-1}$, increment: $21 \mathrm{~K} \mathrm{~km} \mathrm{~s}^{-1}$, rms noise: $0.91 \mathrm{~K} \mathrm{~km} \mathrm{~s}^{-1}$ ). e) $\mathrm{C}^{18} \mathrm{O} J=1-0$ (lowest contour: $0.5 \mathrm{~K} \mathrm{~km} \mathrm{~s}^{-1}$, increment: $2 \mathrm{~K} \mathrm{~km} \mathrm{~s}^{-1}$, rms noise: $0.52 \mathrm{~K} \mathrm{~km} \mathrm{~s}^{-1}$ ). f) $\mathrm{C}^{17} \mathrm{O} J=1-0$ (lowest contour: $0.1 \mathrm{~K} \mathrm{~km} \mathrm{~s}^{-1}$, increment: $0.5 \mathrm{~K} \mathrm{~km} \mathrm{~s}^{-1}$, rms noise: $0.14 \mathrm{~K} \mathrm{~km} \mathrm{~s}^{-1}$ ). g) $\mathrm{HCN} J=1-0$ (lowest contour: $14 \mathrm{~K} \mathrm{~km} \mathrm{~s}^{-1}$, increment: $7 \mathrm{~K} \mathrm{~km} \mathrm{~s}^{-1}$, rms noise: $1.04 \mathrm{~K} \mathrm{~km} \mathrm{~s}^{-1}$ ). h) $\mathrm{HCO}^{+} J=1-0$ (lowest contour: $10 \mathrm{~K} \mathrm{~km} \mathrm{~s}^{-1}$, increment: $4.5 \mathrm{~K} \mathrm{~km} \mathrm{~s}^{-1}$, rms noise: $0.78 \mathrm{~K} \mathrm{~km} \mathrm{~s}^{-1}$ ). feature common to all molecular lines observed by us across the eastern part of the FIR II region.

Line intensity contrasts are high. Ratios of integrated intensity between the position of strongest emission in the mapped area (offset relative to the position of minimum emission: $\left.(\Delta \alpha, \Delta \delta)=\left(60^{\prime \prime}, 80^{\prime \prime}\right)\right)$ and the minimum itself at $\alpha_{1950}=$ $17^{\mathrm{h}} 17^{\mathrm{m}} 26^{\mathrm{s}} .7, \delta_{1950}=-35^{\circ} 46^{\prime} 10^{\prime \prime}\left(\alpha_{2000}=17^{\mathrm{h}} 20^{\mathrm{m}} 47^{\mathrm{s}} .9, \delta_{2000}=\right.$ $\left.-35^{\circ} 49^{\prime} 08^{\prime \prime}\right)$ are $4,10,6,4,6$, and 6 for $\mathrm{CO},{ }^{13} \mathrm{CO} J=1-0$, ${ }^{13} \mathrm{CO} \mathrm{J}=2-1, \mathrm{C}^{18} \mathrm{O}, \mathrm{HCN}$, and $\mathrm{HCO}^{+}$, respectively (because of its weakness, $\mathrm{C}^{17} \mathrm{O}$ is not included here; the error in the ratios can be deduced from the $15 \%$ calibration uncertainties given in Sect. 2.1 and is therefore of order $\pm 20 \%$ ). The ratios are lower limits because not only the main beam but also the sidelobes may contribute to the signal seen at the center of the molecular void. The size of the region is $1{ }^{\prime} .5 \pm 0,2$, if measured where integrated intensities reach twice the minimum value. This holds for $\mathrm{CO}$, for the rare $\mathrm{CO}$ isotopomers, as well as for the high density tracers $\mathrm{HCN}$ and $\mathrm{HCO}^{+}$. The size is significantly larger than the beam (Sect. 2.1), so that estimates based on ${ }^{13} \mathrm{CO} J=1-0$ and $2-1$ data (FWHP beamwidths: $45^{\prime \prime}$ and $24^{\prime \prime}$ ) do agree even without beam deconvolution. While in $\mathrm{CO}$ the center of the molecular void is surrounded by a closed ring of strong $\mathrm{CO}$ emission, the rare $\mathrm{CO}$ isotopomers show an opening of the ring toward the south. The high density tracers $\mathrm{HCN}$ and $\mathrm{HCO}^{+}$also show such an opening toward the south-east and east. Here only half of the ring is prominent, even though the minimum is clearly identified. From this we conclude that molecular column densities are particularly high in the east, north, and west, while densities reach the highest values near the northern and western edges of the molecular void.

Calculating the mean velocity (first moment) of the observed emission, we show the velocity distribution of the ${ }^{13} \mathrm{CO} \mathrm{J}=$ 1-0 line in Fig. 2. The other lines show a similar distribution. Molecular gas with "low" velocities dominates the northeast (down to $-6.5 \mathrm{~km} \mathrm{~s}^{-1}$ ), while gas with "high" velocities is observed in the southwest (up to $-2 \mathrm{~km} \mathrm{~s}^{-1}$ ). The velocity gradient is approximately $5 \mathrm{~km} \mathrm{~s}^{-1} \mathrm{pc}^{-1}$; linewidths are $\sim 5-6 \mathrm{~km} \mathrm{~s}^{-1}$.

\subsection{Atomic carbon emission}

To study the neutral atomic component of the molecular void, we observed the two [CI] lines of the ${ }^{3} \mathrm{P}$ fine structure system at $492\left({ }^{3} \mathrm{P}_{1}-{ }^{3} \mathrm{P}_{0}\right)$ and $809 \mathrm{GHz}\left({ }^{3} \mathrm{P}_{2}-{ }^{3} \mathrm{P}_{1}\right)$. [C $\left.\mathrm{I}\right]$ is considered to be a classical PDR tracer (e.g., Hollenbach \& Tielens 1997). The cross consisting of 19 positions along the E-W and N-S directions is shown in Fig. 3. The spacing is $10^{\prime \prime}$. While one might have expected particularly strong emission at the inner portion of the molecular shell, with weaker emission deeper inside the molecular cloud, this is not seen. Instead, the two atomic carbon 


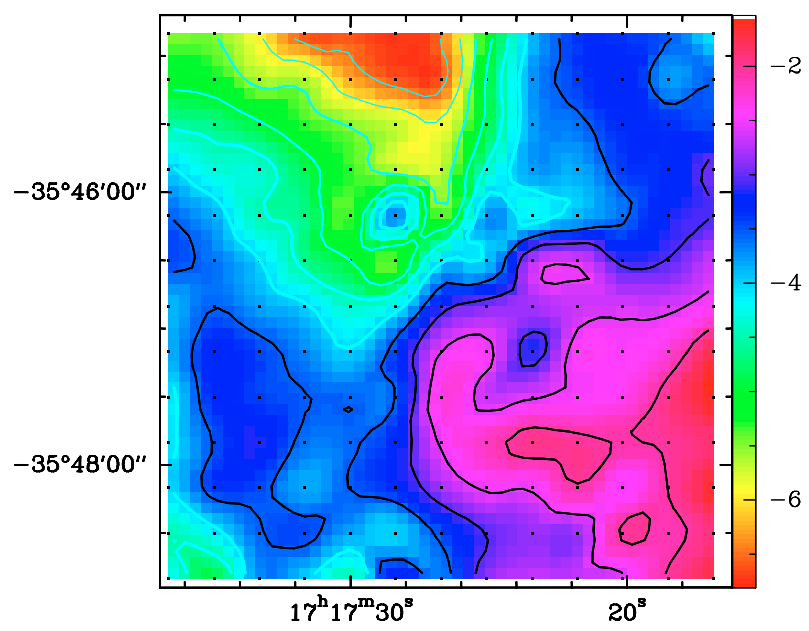

Fig. 2. Local standard of rest $\left(V_{\mathrm{LSR}}\right)$ velocity distribution of ${ }^{13} \mathrm{CO}(1-0)$ across the eastern part of NGC 6334 FIR II (B1950.0 coordinates). Contours: -6.5 to $-2.0 \mathrm{~km} \mathrm{~s}^{-1}$ by $0.5 \mathrm{~km} \mathrm{~s}^{-1}$. $1^{\prime}$ corresponds to $0.5 \mathrm{pc}$.

lines show a trend that is similar to that of the molecular emission lines. The line intensity increases when leaving the inner region of the void, reaching a high plateau where the surrounding molecular cloud is located.

As in the case of the rare $\mathrm{CO}$ isotopomers, the molecular edge toward the south exhibits weaker emission than the positions at the other ends of the cross. Because of the much smaller beamsize of the $[\mathrm{CI}]$ measurements when being compared with the molecular data (Sect. 3.1), the size of the region devoid of strong emission as well as spatial fine structure can be evaluated with higher accuracy. Along the E-W axis, we find an extent of $80^{\prime \prime}$ for the molecular void; along the N-S axis the corresponding value is $100^{\prime \prime}$, defined in the same way as for the molecular emission in Sect. 3.1. Toward the west, the edge of the void is particularly pronounced. Here the $[\mathrm{CI}]$ emission rises by a factor of $>2$ within $10^{\prime \prime}(0.1 \mathrm{pc})$. This is the region that appears to be characterized by high column densities and high particle densities (Sect. 3.1). A significant difference between the distributions of the 492 and $809 \mathrm{GHz}$ lines of [C I] is not apparent. Within the limits of spatial resolution, the morphology of [C I] is compatible with that of the molecular tracers.

For the central position, the main beam brightness temperatures are $\sim 4$ and $\sim 6 \mathrm{~K}$ for $[\mathrm{CI}]{ }^{3} \mathrm{P}_{1}-{ }^{3} \mathrm{P}_{0}$ and ${ }^{3} \mathrm{P}_{2}-{ }^{3} \mathrm{P}_{1}$, respectively, while temperatures of order $20-30 \mathrm{~K}$ are reached in the outermost eastern and western positions. The contrast between the molecular edge and the central emission features is $\sim 5 \pm 1$. This is consistent with the corresponding molecular ratios choosing the same reference positions. While the mean velocities agree with those of the molecular lines (see Fig. 2), the [C I] lines are slightly narrower, typically $\sim 4 \mathrm{~km} \mathrm{~s}^{-1}$. This is likely caused by the different beamwidths (Table 1).

\section{Analysis and discussion}

In the following we give a brief overview on NGC 6334 with an emphasis on FIR II. Then physical parameters of the gas near the void are evaluated, starting with an analysis of [CI] that is followed by $\mathrm{CO}$ and the high density tracers $\mathrm{HCN}$ and $\mathrm{HCO}^{+}$. After searching for traces of molecular outflows seen in $\mathrm{CO}$, ${ }^{18} \mathrm{O} /{ }^{17} \mathrm{O}$ ratios and the evolutionary stage of the entire region are also discussed.

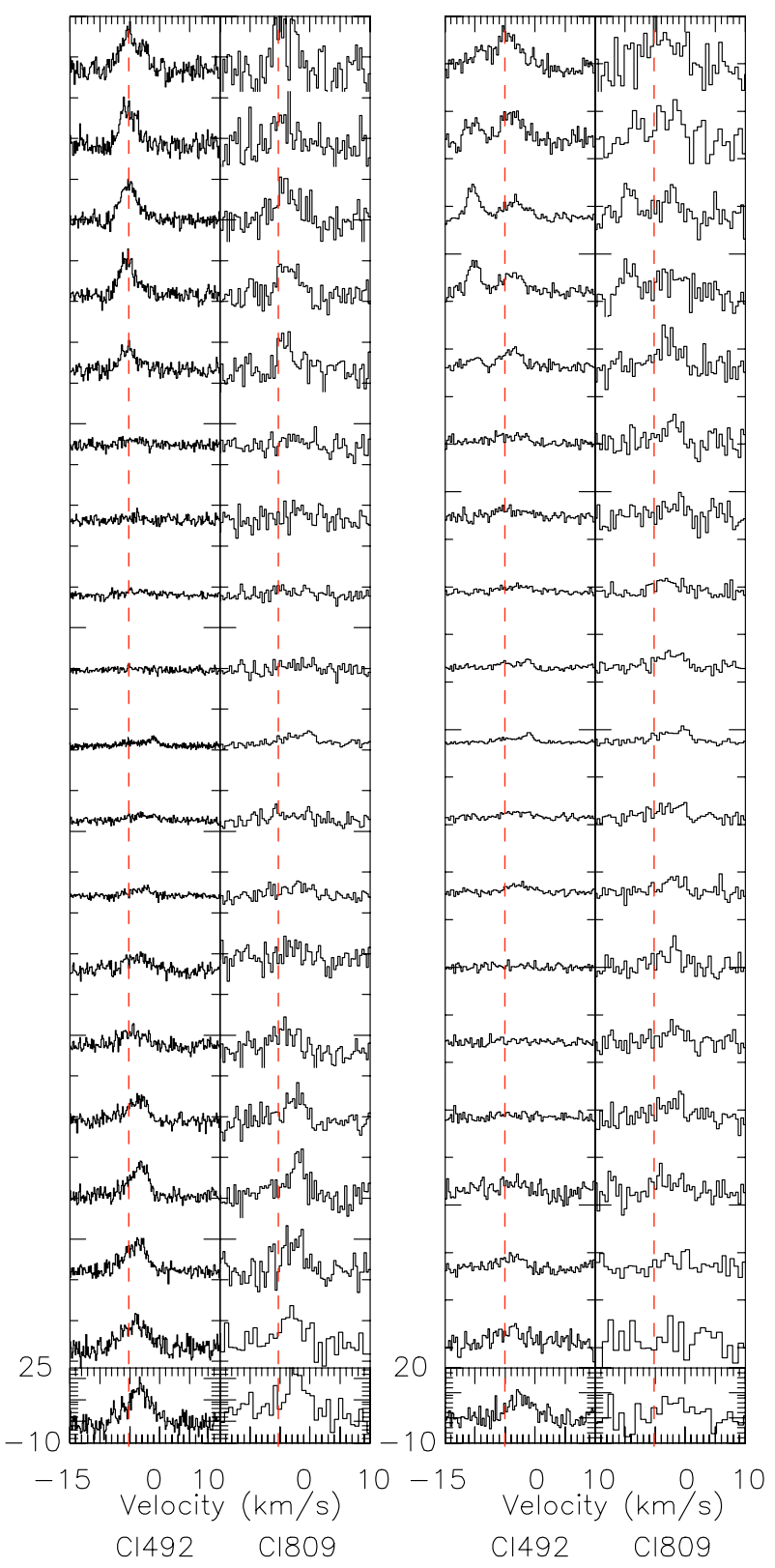

Fig. 3. Spectra of the $[\mathrm{CI}]{ }^{3} \mathrm{P}_{1}-{ }^{3} \mathrm{P}_{0} 492 \mathrm{GHz}$ line (channel spacings $0.15 \mathrm{~km} \mathrm{~s}^{-1}$ (left panel) and $0.30 \mathrm{~km} \mathrm{~s}^{-1}$ (right panel)) and the ${ }^{3} \mathrm{P}_{2}-{ }^{3} \mathrm{P}_{1} \quad 809 \mathrm{GHz}$ line (channel spacings $0.68 \mathrm{~km} \mathrm{~s}^{-1}$ (left panel) and $0.90 \mathrm{~km} \mathrm{~s}^{-1}$ (right panel)) toward the molecular void region. In the left panel, west is up and east is down; in the right one north is up and south is down. The central position is at $\alpha_{1950}=17^{\mathrm{h}} 17^{\mathrm{m}} 26^{\mathrm{s}} .7$, $\delta_{1950}=-35^{\circ} 46^{\prime} 10^{\prime \prime}\left(\alpha_{2000}=17^{\mathrm{h}} 20^{\mathrm{m}} 47^{\mathrm{s}} .9, \delta_{2000}=-35^{\circ} 49^{\prime} 08^{\prime \prime}\right)$, the spacing is $10^{\prime \prime}$. The ordinate is in units of main-beam brightness temperature $(\mathrm{K})$. To facilitate comparisons, $V_{\mathrm{LSR}}=-5 \mathrm{~km} \mathrm{~s}^{-1}$ is marked by dashed vertical lines. Adopted rest frequencies are 492.160651 and 809.341970 GHz.

\subsection{NGC 6334: a brief overview}

NGC $6334^{2}$ hosts some of the most active sites of star formation in the Galaxy. Embedded in an extended region of $\mathrm{H} \alpha$ emission that encompasses almost $1^{\circ}$ (Meaburn \& White 1982;

2 The nomenclature of molecular, maser, and infrared features is quite complex (see the appendix in Kuiper et al. 1995). We follow recent publications in using the nomenclature given by McBreen et al. (1979) and Gezari (1982) for the infrared sources and Rodríguez et al. (1982) for the H II regions. 


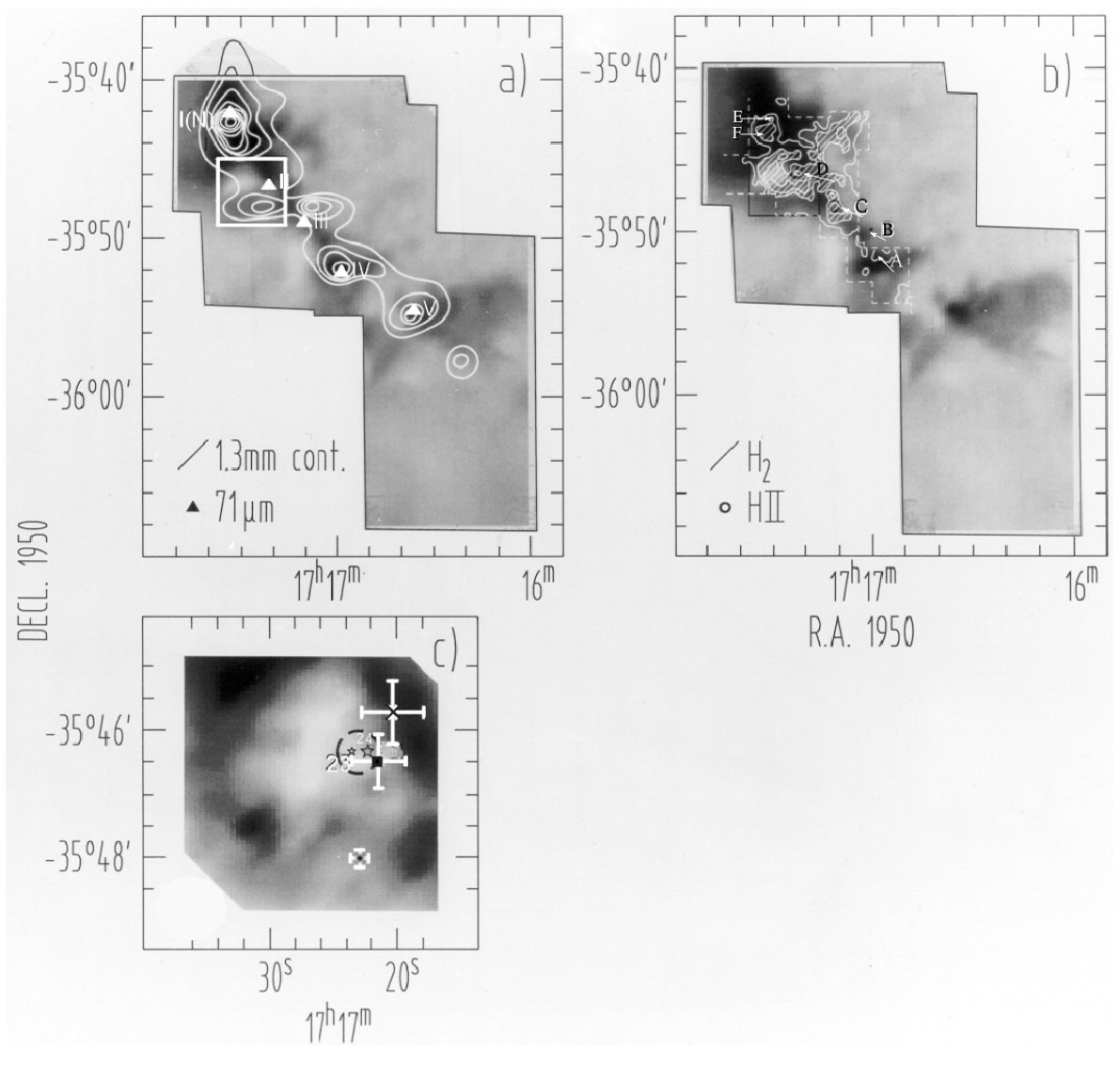

Fig. 4. Finder charts of the features on a larger $(a, b)$ and smaller (c) scale toward NGC 6334. The inset in a) (white) and b) (black) shows the region sketched in more detail in c). The grey scale outlines the integrated ${ }^{13} \mathrm{CO} J=1-0$ intensity (this paper). a) Contours show the distribution of the $1 \mathrm{~mm}$ continuum emission (Gezari \& Blitz 1990). Triangles with roman numbers mark the positions of the $71 \mu \mathrm{m}$ peaks (Loughran et al. 1986). b) White contours show the distribution of shocked $\mathrm{H}_{2}$ gas (Straw \& Hyland 1989b), with regions of strongest emission being emphasized by white diagonal lines. Prominent $\mathrm{H}$ II regions are marked by $\mathrm{A}, \mathrm{B}, \mathrm{C}, \mathrm{D}, \mathrm{E}, \mathrm{F}$ (see Rodríguez et al. 1982). c) The Strömgren sphere of the $\mathrm{H}$ II region $\mathrm{D}$ is shown as a black dashed ellipse for $n\left(\mathrm{H}_{2}\right)=10^{4} \mathrm{~cm}^{-3}\left(R_{\text {str }} \propto\right.$ $n\left(\mathrm{H}_{2}\right)^{-2 / 3}$; see Sect. 4.8). The two $K$-band stars IRS-23 (early B) and IRS-24 (likely O6.5) are given as smaller and larger black asterisks, respectively (e.g., Straw et al. 1989). The $1 \mathrm{~mm}$ peak identified by Gezari \& Blitz (1990) is plotted as a small dot in the southwestern part of the map. The $42 \mu \mathrm{m}$ FIR peak (Loughran et al. 1986) is marked as a black square with white error bars and the $\mathrm{H}_{2} \mathrm{O}$ maser identified by Moran \& Rodríguez (1980) is displayed as a cross, also with white error bars, near the northwestern edge of the map.
Burton et al. 2000) lies a giant molecular cloud (GMC). Its ridge of length $\sim 30^{\prime}$ at $\mathrm{PA} \sim 45^{\circ}$ (see Figs. 1a and 4a for ${ }^{13} \mathrm{CO} J=1-0$ emission) shows spectacular, complex structure including dense molecular clumps, maser sources, H II regions, and luminous sources of dust emission (Fig. 4). The ridge has been detected in many energy bands ranging from low frequency radio waves up to $80 \mathrm{keV}$ (Burton et al. 2000; Caproni et al. 2000; Kraemer et al. 2000; McCutcheon et al. 2000; Persi et al. 2000; Sandell 2000; Sarma et al. 2000; Brooks \& Whiteoak 2001; Carral et al. 2002; Bykov et al. 2006; Ezoe et al. 2006; Hunter et al. 2006; Leurini et al. 2006; Schilke et al. 2006, and references therein).

Along the active ridge, NGC 6334 FIR II is the outstanding source of far infrared emission $\left(L_{\mathrm{FIR}} \sim 3 \times 10^{5} L_{\odot}\right.$; see Fig. $\left.4 \mathrm{c}\right)$. Its peak shifts with increasing wavelength to the west, with the $21 \mu \mathrm{m}$ and $134 \mu \mathrm{m}$ peaks being displaced by about $2^{\prime}$ (Loughran et al. 1986). Slightly southeast of the infrared peak lies an H II region (NGC 6334-D) of diameter 2' that is known to show an almost circular, featureless thermal radio continuum distribution (Brooks \& Whiteoak 2001 and references therein) and that is characterized by an exceptionally low extinction (the "molecular void"; see Straw \& Hyland 1989a for an extinction map). This H II region appears to be powered by a highly obscured O6.5 star, possibly the most massive star of the entire GMC (Neckel 1978; Rodríguez et al. 1982; Loughran et al. 1986; Straw et al. 1989).

Straw \& Hyland (1989b) imaged the $\mathrm{H}_{2} 2.12 \mu \mathrm{m} v=$ 1-0 S(1) line that shows an intense arc at the southern, eastern, and northern edges of the radio continuum source (see also Fig. 4b) being part of a ring surrounding the H II region. In addition, they took near infrared broad band spectra toward selected positions. Analyzing line ratios they conclude that most of the $\mathrm{H}_{2}$ emission is caused by shocks rather than by fluorescence triggered by UV radiation. However, extinction is severely affecting measured line ratios, so that the case is not entirely clear (see also Burton et al. 2000). The good correlation between this $\mathrm{H}_{2}$ ring and the dust extinction led Straw \& Hyland (1989b) to propose that young stars have swept the inner part of the ring (the molecular void) free of gas and dust and that the $\mathrm{H}_{2}$ emission is powered by the interaction between the stellar wind and the remaining molecular gas. The shell is also seen in the $3.3 \mu \mathrm{m}$ emission of polycyclic aromatic hydrocarbon (PAH) (Burton et al. 2000). Overall, FIR II appears to belong to the more evolved centers of activity in NGC 6334 because the far infrared source and the associated $\mathrm{H}$ II region are more extended than other similar targets in the region (Loughran et al. 1986; Straw et al. 1989) and because there is no indication of particularly low molecular densities in the surrounding environment (e.g., Sect. 3.1).

\subsection{Global distribution of the molecular gas}

The distribution of our large-scale ${ }^{13} \mathrm{CO} J=1-0$ map (Fig. 1a) agrees well with the $\mathrm{CO} J=2-1$ and $\mathrm{CS} J=3-2$ maps presented by Kraemer \& Jackson (1999). Our ${ }^{13} \mathrm{CO} J=$ 1-0 map shows pronounced peaks toward NGC 6334 FIR I (up to $\sim 150 \mathrm{~K} \mathrm{~km} \mathrm{~s}^{-1}$ ), IV and V, while FIR II and III are associated with tongues of ridge emission extending southwards from FIR I and northeastwards from FIR IV, respectively. The less well sampled southwestern part of our ${ }^{13} \mathrm{CO}$ map, not covered by Kraemer \& Jackson (1999), shows no significant additional features. For the narrow ridge, our map is also consistent with the ${ }^{13}$ CO $J=2-1$ map of Kraemer \& Jackson (1999; their Fig. 3) that covers a smaller region. 
Table 2. [CI] excitation parameters for five key positions (center and outermost positions toward E, S, W, and N; see Fig. 3)*

\begin{tabular}{|c|c|c|c|c|c|c|c|c|c|}
\hline \multirow{3}{*}{ Pos. } & \multicolumn{2}{|c|}{$T_{\mathrm{mb}} \mathrm{d} v$} & \multicolumn{2}{|c|}{$T_{\mathrm{mb}}$} & \multirow[t]{3}{*}{$R$} & \multirow{3}{*}{$\begin{array}{l}T_{\mathrm{ex}} \\
(\mathrm{K})\end{array}$} & \multirow{3}{*}{$\tau_{1-0}$} & \multirow{3}{*}{$\tau_{2-1}$} & \multirow{3}{*}{$\begin{array}{c}N_{\mathrm{CI}} \\
\left(10^{17} \mathrm{~cm}^{-2}\right)\end{array}$} \\
\hline & $(1-0)$ & $(2-1)$ & $(1-0)$ & $(2-1)$ & & & & & \\
\hline & & $(\mathrm{K}$ & & & & & & & \\
\hline$(0,0)$ & $14.3 \pm 1.7$ & $28.2 \pm 4.8$ & $4.1 \pm 1.0$ & $6.5 \pm 1.7$ & $1.97 \pm 0.30$ & $\gtrsim 200$ & $\lessgtr 0.02$ & $\lessgtr 0.04$ & $\sim 2$ \\
\hline $\mathrm{E}$ & $80.9 \pm 3.9$ & $79.2 \pm 13.5$ & $18.6 \pm 4.7$ & $27.1 \pm 6.8$ & $0.98 \pm 0.15$ & $51 \pm 13$ & $0.6(+0.5,-0.2)$ & $\gtrsim 0.8$ & $\sim 15$ \\
\hline $\mathrm{W}$ & $70.3 \pm 3.7$ & $72.1 \pm 13.6$ & $18.1 \pm 4.5$ & $26.6 \pm 6.7$ & $1.02 \pm 0.15$ & $53 \pm 9$ & $0.6(+0.2,-0.1)$ & $1.3(+2.2,-0.4)$ & $\sim 13$ \\
\hline $\mathrm{N}$ & $92.4 \pm 4.6$ & $113.3 \pm 13.9$ & $16.5 \pm 4.5$ & $21.0 \pm 5.3$ & $1.23 \pm 0.18$ & $72 \pm 20$ & $0.3(+0.2,-0.1)$ & $0.5(+0.4,-0.2)$ & $\sim 15$ \\
\hline S & $40.6 \pm 4.5$ & $39.2 \pm 14.0$ & $11.0 \pm 2.8$ & $13.7 \pm 3.4$ & $0.96 \pm 0.14$ & $49 \pm 10$ & $0.3(+0.2,-0.1)$ & $0.6(+0.3,-0.2)$ & $\sim 6$ \\
\hline
\end{tabular}

* Columns:

Column 1: offset position; E, W, N, and S denote the outermost positions shown in Fig. 3, with offsets of 90" along the four cardinal directions. Columns 2 and 3: integrated main beam brightness temperatures of the ${ }^{3} \mathrm{P}_{1}-{ }^{3} \mathrm{P}_{0}$ and ${ }^{3} \mathrm{P}_{2}-{ }^{3} \mathrm{P}_{1}$ lines; errors are determined from Gaussian fits.

Columns 4 and 5: main beam brightness temperatures of the ${ }^{3} \mathrm{P}_{1}-{ }^{3} \mathrm{P}_{0}$ and ${ }^{3} \mathrm{P}_{2}-{ }^{3} \mathrm{P}_{1}$ lines; errors reflect uncertainties in absolute calibration ( $\pm 25 \%$; see Sect. 2) and are not providing information on signal-to-noise ratios.

Column 6: integrated intensity ratio $\left([\mathrm{CI}]{ }^{3} \mathrm{P}_{2}-{ }^{3} \mathrm{P}_{1} /[\mathrm{CI}]{ }^{3} \mathrm{P}_{1}-{ }^{3} \mathrm{P}_{0}\right)$ with $15 \%$ uncertainties as estimated in Sect. 2.

Column 7: excitation temperature, derived from $T_{\mathrm{ex}}=38.8 / \ln (2.11 / R)($ Schneider et al. 2003; for $R$, see Col. 6).

Columns 8 and 9: optical depths, derived from $\tau_{1-0}=-\ln \left(1-T_{\mathrm{mb}}(1-0) \times\left(\exp \left(23.62 / T_{\mathrm{ex}}\right)-1\right) / 23.62\right)$ and $\tau_{2-1}=-\ln \left(1-T_{\mathrm{mb}}(2-1) \times\left(\exp \left(38.8 / T_{\mathrm{ex}}\right)-\right.\right.$ 1)/38.8) (Schneider et al. 2003).

Column 10: [CI] column density, derived from $N_{\mathrm{CI}}=5.94 \times 10^{15} \times \tau_{1-0} /\left(1-\exp \left(-\tau_{1-0}\right)\right) \times\left[\left(1+3 \exp \left(-23.6 / T_{\operatorname{ex}}\right)+\right.\right.$ $\left.\left.5 \exp \left(-62.4 / T_{\mathrm{ex}}\right)\right) / 3 \exp \left(-23.6 / T_{\mathrm{ex}}\right)\right] \times \int T_{\mathrm{mb}}(1-0) \mathrm{d} v$ (see Schneider et al. 2003).

\section{3. [CI] excitation, optical depth, column density, and correlation with ${ }^{13} \mathrm{CO}$}

The C I triplet with lines near 492 and $809 \mathrm{GHz}$ is a useful tracer of excitation. Our measured $[\mathrm{CI}]{ }^{3} \mathrm{P}_{2}-{ }^{3} \mathrm{P}_{1}$ brightness temperatures are slightly higher than those of the $[\mathrm{CI}]{ }^{3} \mathrm{P}_{1}-{ }^{3} \mathrm{P}_{0}$ line (see below). This is consistent with results from other galactic star-forming regions (e.g., Schneider et al. 2003). Obtaining Gaussian fits to the $[\mathrm{CI}]$ spectra, we calculated integrated line intensities for five key positions, the central one and the four outermost positions along the four cardinal directions (see Fig. 3). Assuming local thermodynamic equilibrium (LTE), optically thin line emission, and uniform cloud coverage, the integrated intensity ratios allow us to determine the excitation temperature and optical depths of both transitions as well as the beam averaged C I column density (e.g., Stutzki et al. 1997; Weiß et al. 2003). Table 2 displays the resulting parameters and the equations used. The ratios of integrated line intensities $(\sim 1.2-1.5)$ and excitation temperatures $(50-70 \mathrm{~K})$ are similar for the four edge positions. Toward the central position of the molecular void, however, the line ratio is almost two and the excitation temperature must be $\gtrsim 200 \mathrm{~K}$. This is in accordance with the temperature of the photodissociated gas assumed by Kraemer et al. (2000), $250 \mathrm{~K}$. While in view of possible systematic calibration errors the exact value given in Table 2 may be debatable, we note that the integration time toward the central position is much higher than toward all other positions, so that a low signal-tonoise ratio does not affect the deduced parameters. Furthermore, all adjacent positions still belonging to the inner part of the void confirm, albeit with lower signal-to-noise ratios, the high $[\mathrm{CI}]{ }^{3} \mathrm{P}_{2}-{ }^{1} \mathrm{P}_{0} /{ }^{3} \mathrm{P}_{1}-{ }^{3} \mathrm{P}_{0}$ line intensity ratio. Since the [CI] lines are likely thermalized ( $\left.T_{\mathrm{ex}} \sim T_{\mathrm{kin}}\right)$, we conclude that the inner part of the studied region is dominated by gas with temperatures that are much higher than those in the predominantly molecular outer regions. While the $[\mathrm{CI}]$ optical depth at the center of the void is one to two orders of magnitude below those at the molecular edges, the total C I column density is as a consequence of the high excitation, only a factor of several smaller.

With respect to intensity and morphology, the 492 and $809 \mathrm{GHz}$ lines of neutral carbon are known to be correlated with low-J CO emission (e.g., Ikeda et al. 2002). The $\left[\mathrm{C} I{ }^{3} \mathrm{P}_{1}-{ }^{3} \mathrm{P}_{0}\right.$ and ${ }^{3} \mathrm{P}_{2}-{ }^{3} \mathrm{P}_{1}$ fine structure lines have upper energy
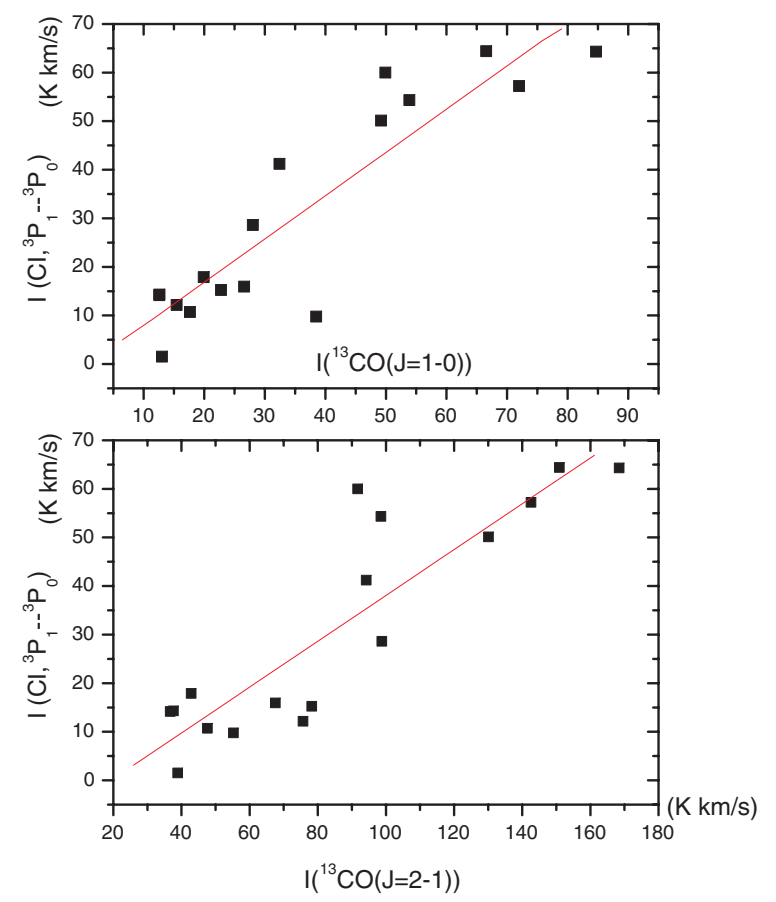

Fig. 5. A comparison of integrated intensities between the $[\mathrm{C} I]{ }^{3} \mathrm{P}_{1}-{ }^{3} \mathrm{P}_{0}$ and the ${ }^{13} \mathrm{CO} J=1-0$ and $2-1$ lines.

levels of $23.6 \mathrm{~K}$ and $62.5 \mathrm{~K}$ above the ground state, respectively. Critical densities are of order $10^{3}$ and $10^{4} \mathrm{~cm}^{-3}$ for collisions with $\mathrm{H}_{2}$. These critical densities agree with those of the lowest rotational transitions of optically thin $\mathrm{CO}$ isotopomers (e.g., Mao et al. 2000). Toward the molecular void, main beam brightness temperatures of the $[\mathrm{CI}]{ }^{3} \mathrm{P}_{1}-{ }^{3} \mathrm{P}_{0}$ line are similar to those of the ${ }^{13} \mathrm{CO} J=1-0$ transition and are about a factor of $\sim 1.6$ lower than those of ${ }^{13} \mathrm{CO} J=2-1$. Correlations between ${ }^{13} \mathrm{CO}$ and [C I] emission are shown in Fig. 5. Ignoring differences in beam size we compare the integrated intensities for the 17 positions with both ${ }^{13} \mathrm{CO}$ and [C I] emission measured. Results from linear fitting are $I\left(\mathrm{CI}\left({ }^{3} \mathrm{P}_{1}-{ }^{3} \mathrm{P}_{0}\right)\right)=(0.89 \pm 0.11) I\left({ }^{13} \mathrm{CO}(J=1-0)\right)+$ $(-1.00 \pm 4.67)$ and $I\left(\mathrm{CI}\left({ }^{3} \mathrm{P}_{1}-{ }^{3} \mathrm{P}_{0}\right)\right)=(0.47 \pm 0.06) I\left({ }^{13} \mathrm{CO}(J=\right.$ $2-1))+(-9.12 \pm 6.10)$, with correlation coefficients of $r=0.88$ and $r=0.90$, respectively. Again, this is consistent with results 


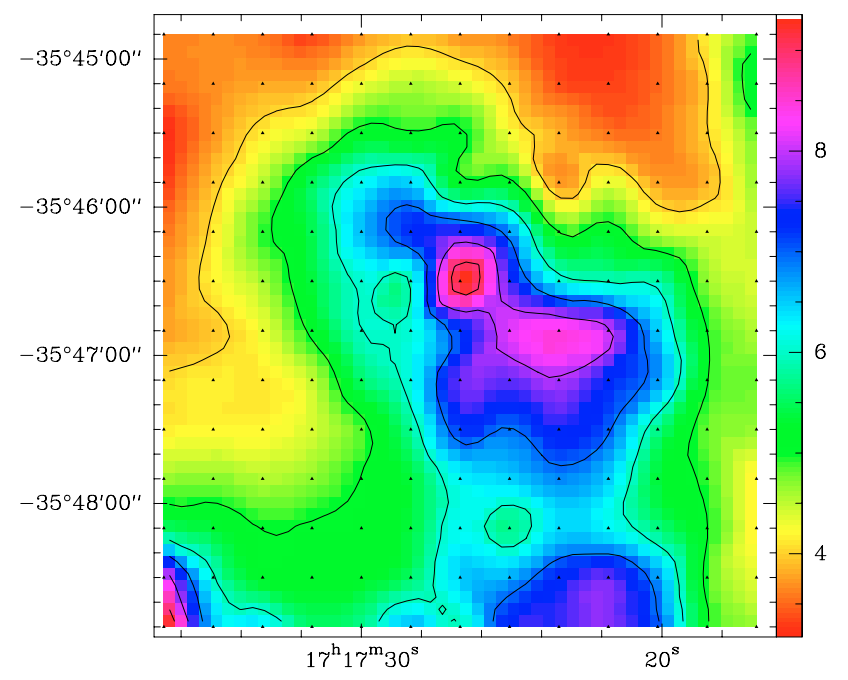

Fig. 6. Integrated ${ }^{12} \mathrm{CO} /{ }^{13} \mathrm{CO} J=1-0$ line intensity ratios near N 6334-D. Contours: 4-9, increment: 1.

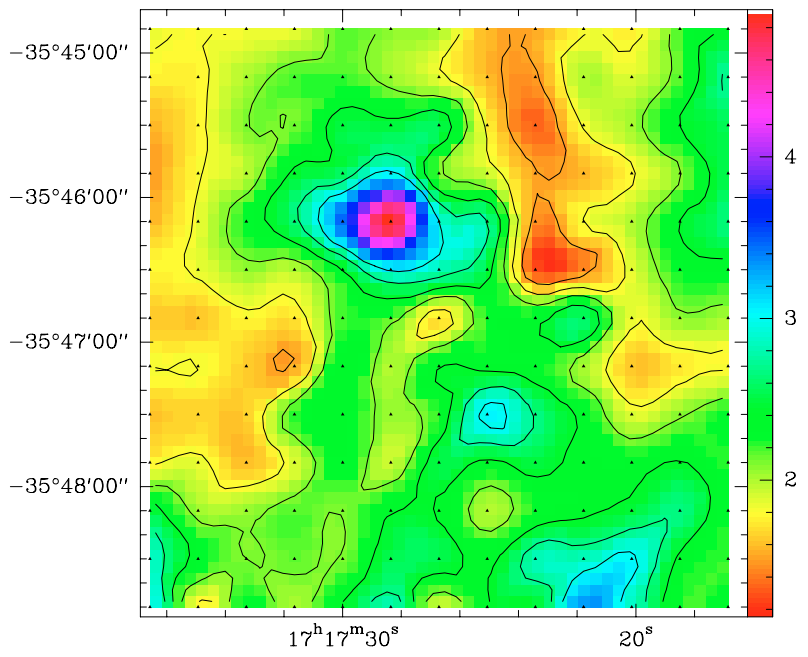

Fig. 7. Integrated ${ }^{13} \mathrm{CO} J=2-1 / J=1-0$ line intensity ratios near NGC 6334-D. Contours: 1.5-3.0, increment: 0.3.

from other molecular clouds (e.g., Plume et al. 1999; Ikeda et al. 2002).

\section{4. $\mathrm{CO}$}

Not only atomic but also molecular lines can be a measure of excitation conditions. Figures 6 and 7 show the integrated $\mathrm{CO} /{ }^{13} \mathrm{CO} J=1-0$ and ${ }^{13} \mathrm{CO} J=2-1 / J=1-0$ line ratios for the area covering the molecular void. ${ }^{12} \mathrm{CO} /{ }^{13} \mathrm{CO}$ ratios peak, not surprisingly, at the very center of the map where the expanding $\mathrm{H}$ II region has removed most of the molecular gas along the line of sight. Here the line ratios almost reach a value of 10 , while values of $\sim 4$ characterize the partial ring of relatively high column density that opens up toward the south (see also Sect. 3.1).

$\mathrm{CO}$ must be optically thick because $\mathrm{CO} /{ }^{13} \mathrm{CO}$ line intensity ratios are much smaller than the "local" ${ }^{12} \mathrm{C} /{ }^{13} \mathrm{C}$ ratio of $\sim 70$ (e.g., Wilson \& Rood 1994). From the peak CO temperature, assuming uniform coverage of the beam, the $\mathrm{CO}$ excitation temperature $T_{\text {ex }}$ can then be estimated by

$T_{\mathrm{mb}, \mathrm{CO}}=\frac{h v}{k}\left[\left(\mathrm{e}^{h v / k T_{\mathrm{ex}}}-1\right)^{-1}-\left(\mathrm{e}^{h v / k T_{\mathrm{cmb}}}-1\right)^{-1}\right]$.
With $T_{\mathrm{mb}} \sim 40-50 \mathrm{~K}\left(T_{\mathrm{cmb}}=2.73 \mathrm{~K}\right)$ in the molecular cloud surrounding the void we get $T_{\mathrm{ex}}$ values that are of the same order as $T_{\mathrm{mb}}$. CO must be thermalized because high optical depths reduce the low critical densities even below the values mentioned in Sect. 4.3. Being thermalized and optically thick, the measured $T_{\mathrm{mb}, \mathrm{CO}}$ values provide an estimate of the kinetic temperature of the molecular gas. $T_{\text {kin }} \sim 40-50 \mathrm{~K}$. In the case of self-absorption, we would have only obtained lower limits to $T_{\mathrm{ex}}$ and $T_{\text {kin. }}$. The CO profiles (Fig. A.1) show, however, no evidence for this effect.

In the C I analysis presented in Sect. 4.3 (the E, W, N, and $\mathrm{S}$ positions of Table 2) we obtained similar excitation temperatures as for CO. Does the good agreement between $\mathrm{CI}$ and $\mathrm{CO}$ excitation provide a further argument that $\mathrm{CO}$ selfabsorption can be neglected, that C I may also be thermalized, and that the APEX and SEST beams are approximately uniformly filled with $\mathrm{C}$ I and molecular gas, respectively?

Assuming that (1) the ${ }^{13} \mathrm{CO}$ lines are optically thin, that (2) the cloud covers the beam uniformly, and (3) neglecting the microwave background (a good approximation for $T_{\mathrm{ex}} \gtrsim 10 \mathrm{~K}$ ), a comparison of the ${ }^{13} \mathrm{CO} J=2-1$ and $1-0$ lines provides yet another measure of excitation. Here again line intensity ratios peak near the center of the molecular void, reaching $\sim 4$, while the ratio decreases to $\sim 2$ in the vicinity of the partial ring of high column density (Fig. 7). We then obtain with

$$
T_{\mathrm{ex}, 13 \mathrm{CO}}=-10.6 /\left[\ln \left(\frac{T_{\mathrm{mb}, 2-1}}{4 \times T_{\mathrm{mb}, 1-0}}\right)\right]
$$

excitation temperatures near infinity at the center of the void (within the errors consistent with the high excitation obtained from $\mathrm{CI}$; see the $(0,0)$ position in Table 2$)$ and values $\sim 15 \mathrm{~K}$ near the "molecular ring". These latter temperatures disagree with those obtained from $\mathrm{CO}$ and $\mathrm{C}$ I. Is this due to subthermal excitation of ${ }^{13} \mathrm{CO}$, is it caused by the fact that $\mathrm{C}$ I traces mostly the UV irradiated surfaces of clouds, or is it an optical depth effect? If ${ }^{13} \mathrm{CO}$ is not optically thin, our derived value for $T_{\mathrm{ex}, 13 \mathrm{CO}}$ would only be a lower limit.

Since the critical densities of $\mathrm{CO},{ }^{13} \mathrm{CO}$, and $\mathrm{C}$ I are similar, different excitation temperatures must either be related to different spatial distributions or to optical depth effects. In the case of line saturation, photon trapping slows radiative rates by $\sim \tau^{-1}$, leading to a greater dominance of collisional processes that tend to shift excitation temperatures upwards, toward $T_{\text {kin }}$. With a $\mathrm{CO} /{ }^{13} \mathrm{CO}$ abundance ratio of 70 and line intensity ratios of or$\operatorname{der} 4, \tau(\mathrm{CO} J=1-0) \sim 20$ and $\tau\left({ }^{13} \mathrm{CO} J=1-0\right) \sim 0.3$. Applying an LVG code, with $\mathrm{H}_{2}-\mathrm{CO}$ collision rates from Flower (2001) and an ortho/para $\mathrm{H}_{2}$ abundance ratio of 3 (not a critical parameter), we find a density of $n\left(\mathrm{H}_{2}\right) \sim 10^{3} \mathrm{~cm}^{-3}$ and an optical depth of order $1.5-2.0$ for the ${ }^{13} \mathrm{CO} J=2-1$ line. Thus the ${ }^{13} \mathrm{CO} J=2-1$ line is likely moderately optically thick and the equation for $T_{\mathrm{ex}, 13 \mathrm{CO}}$ indeed provides too low a value. In addition, ${ }^{13} \mathrm{CO}$ is not fully thermalized $\left(T_{\mathrm{ex}, 13 \mathrm{CO}}<T_{\mathrm{ex}, 12 \mathrm{CO}}\right)$.

To summarize, there is evidence that $\mathrm{CI}$ and $\mathrm{CO}$ show excitation temperatures of $T_{\mathrm{ex}} \sim 40-50 \mathrm{~K}$, which is comparable to the kinetic temperature. $N\left({ }^{13} \mathrm{CO}\right) \sim 10^{17} \mathrm{~cm}^{-2}$ and $n\left(\mathrm{H}_{2}\right) \sim 10^{3} \mathrm{~cm}^{-3}$. These values hold, however, only for the parent molecular cloud. Within the molecular void, excitation and kinetic temperatures are far higher but too uncertain to derive reliable (column) densities. According to Straw \& Hyland (1989a; their Fig. 9), $A_{\mathrm{v}}<10^{\mathrm{m}}$ in the void, corresponding to a column density of $N\left(\mathrm{H}_{2}\right)<3 \times 10^{22} \mathrm{~cm}^{-2}$ (Bohlin et al. 1978) or $N\left({ }^{13} \mathrm{CO}\right)<4 \times 10^{16} \mathrm{~cm}^{-2}\left(\left[\mathrm{H}_{2}\right] /[\mathrm{CO}]=10^{4},[\mathrm{CO}] /\left[{ }^{13} \mathrm{CO}\right]=70\right)$. IRS-24, likely the star that is responsible for the molecular void, 


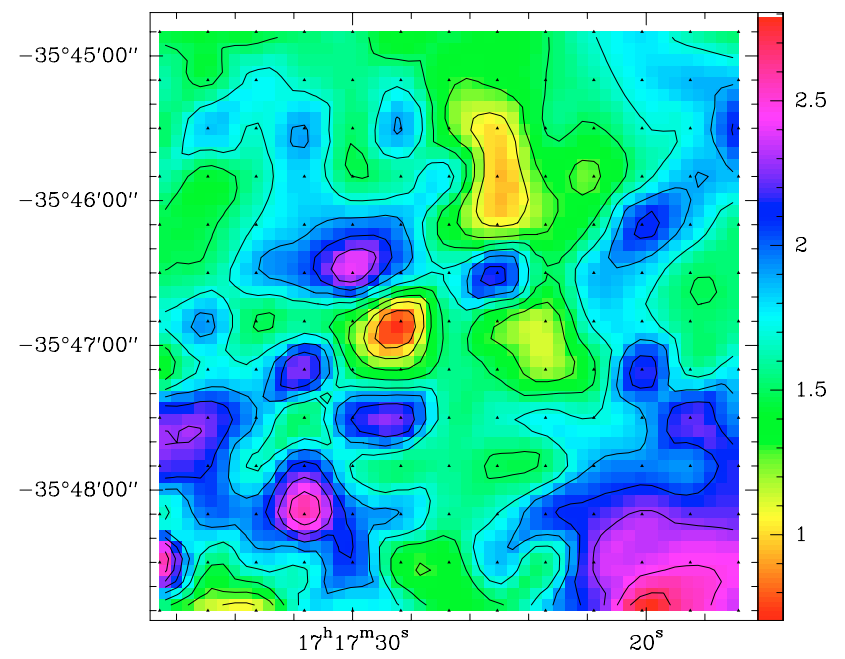

Fig. 8. Integrated $\mathrm{HCN} / \mathrm{HCO}^{+} J=1-0$ line intensity ratios observed with a beam size of $57^{\prime \prime}$ toward NGC 6334-D. Contours: 0.7-2.5, increment: 0.2 .

is reported to show an extinction of $\gtrsim 28^{\mathrm{m}}$ (Straw et al. 1989), corresponding to a ${ }^{13} \mathrm{CO}$ column density of $N\left({ }^{13} \mathrm{CO}\right) \gtrsim$ $10^{17} \mathrm{~cm}^{-2}$. This is compatible with the characteristic value already calculated for the molecular cloud. Apparently, the star is situated near the western edge of the void where column densities are high (IRS-23, a little nearer to the void, shows $A_{\mathrm{v}} \sim 15^{\mathrm{m}}$; Straw et al. 1989). In view of the high obscuration of IRS-24, the star appears to be located behind the bulk of the molecular gas.

\subsection{Other molecules}

The excitation temperatures calculated for $\mathrm{CO}$ and its isotopomers cannot be used for $\mathrm{HCN}$ and $\mathrm{HCO}^{+}$, which have much higher critical densities. An analysis of the HCN hyperfine components shows that the $\mathrm{HCN} J=1-0$ transition, wherever it is strong enough to show its characteristic triple, is optically thin. Adopting $T_{\mathrm{ex}}=10 \mathrm{~K}$ (a reasonable guess implying subthermal excitation), characteristic column densities are of order $N(\mathrm{HCN}) \sim 7 \times 10^{13} \mathrm{~cm}^{-2}$ and $N\left(\mathrm{HCO}^{+}\right) \sim 2 \times 10^{13} \mathrm{~cm}^{-2}$ for the molecular cloud. To reach $T_{\mathrm{mb}}$ values of $5 \mathrm{~K}$ (or more in a few positions), LVG calculations with $T_{\text {kin }}=45 \mathrm{~K}$ and collision rates of Schöier et al. (2005) require $n\left(\mathrm{H}_{2}\right) \gtrsim 10^{5} \mathrm{~cm}^{-3}$. This is a much higher density than that derived from $\mathrm{CO}$, indicating a high degree of small-scale clumping (see also Sect. 4.8). The $\mathrm{HCN} / \mathrm{HCO}^{+} J=1-0$ line intensity ratio is displayed in Fig. 8. In the molecular cloud, $\mathrm{HCN}$ is stronger, in particular in the southwestern region that forms a part of the molecular ridge. Signalto-noise ratios are low for both lines near the void, so that their ratio shows a large scatter. Nevertheless, an inspection of the individual profiles confirms the impression given by Fig. 8, that $\mathrm{HCO}^{+}$becomes about as strong as $\mathrm{HCN}$. This may be a consequence of relatively high kinetic temperatures, strong radiation fields, and low densities near the edge of the molecular cloud (e.g., Fuente et al. 1993; Chin et al. 1997; Brouillet et al. 2005; Christopher et al. 2005).

Averaged integrated intensity ratios are summarized in Table 3 . The intensity ratio of $\mathrm{HCN}$ to $\mathrm{CO}$ could be a qualitative and even a quantitative measure of pressure (Helfer \& Blitz 1997). Our mean values are consistent with observational results from other clouds (e.g., Pirogov 1999; Helfer \& Blitz 1997). Unlike the $\mathrm{HCN}$ and $\mathrm{HCO}^{+} J=1-0$ lines, $\mathrm{CO} J=1-0$ is usually opaque tracing lower density gas. Hence a stronger
Table 3. Average integrated line ratios toward NGC 6334-D ${ }^{a}$.

\begin{tabular}{lr}
\hline \hline Transitions & Ratio \\
\hline$I(\mathrm{CO} J=1-0) / I\left({ }^{13} \mathrm{CO} J=1-0\right)$ & $5.3 \pm 0.1$ \\
$I\left({ }^{13} \mathrm{CO} J=2-1\right) / I\left({ }^{13} \mathrm{CO} J=1-0\right)$ & $2.2 \pm 0.1$ \\
$I(\mathrm{CO} J=1-0) / I(\mathrm{HCN} J=1-0)$ & $7.1 \pm 0.2$ \\
$I(\mathrm{HCN} J=1-0) / I\left(\mathrm{HCO}^{+} J=1-0\right)$ & $1.7 \pm 0.1$ \\
\hline
\end{tabular}

${ }^{a}$ Given errors were obtained from Gaussian fits (standard deviation).
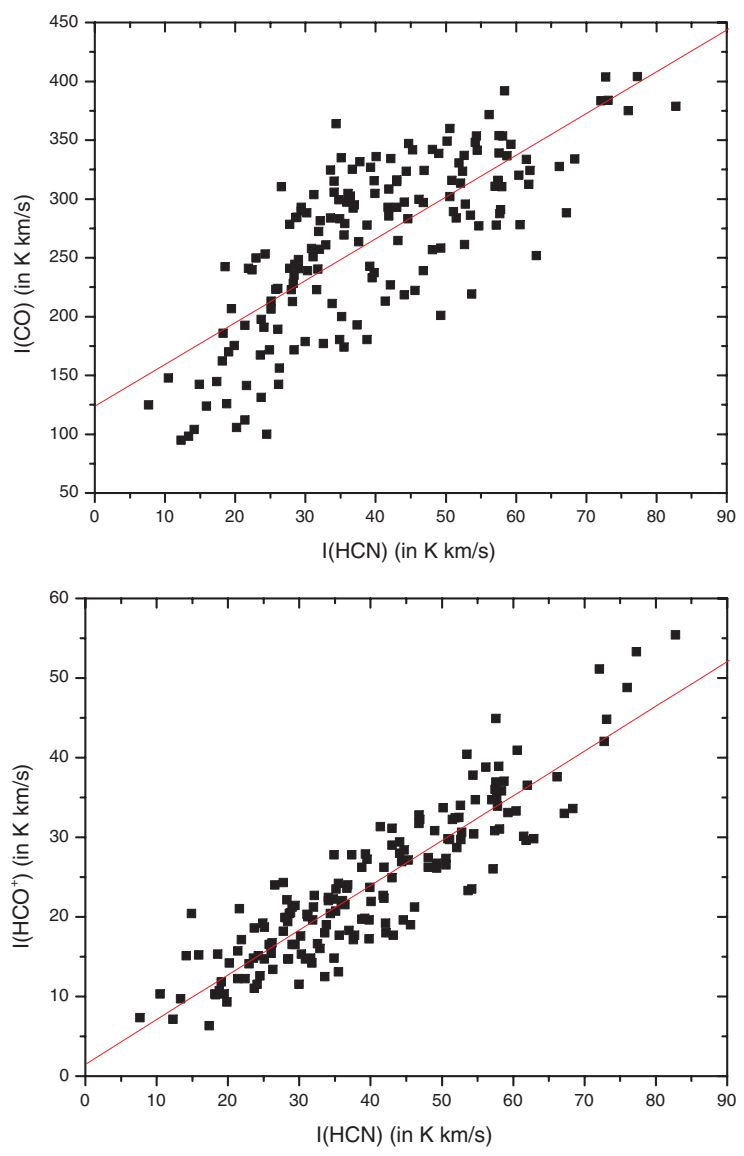

Fig. 9. Comparison of integrated intensites of the $J=1-0$ transitions of $\mathrm{CO}, \mathrm{HCO}^{+}$, and $\mathrm{HCN}$. The lines mark least square fits with $I(\mathrm{CO} J=$ $1-0)=(3.55 \pm 0.24) I(\mathrm{HCN})+(123 \pm 10)$ (correlation coefficient: $0.75)$ and $I\left(\mathrm{HCO}^{+} J=1-0\right)=(0.56 \pm 0.02) I(\mathrm{HCN})+(1.47 \pm 0.89)$ (correlation coefficient: 0.90).

correlation is expected between $\mathrm{HCN}$ and $\mathrm{HCO}^{+}$than between $\mathrm{CO}$ and $\mathrm{HCN}$ or $\mathrm{HCO}^{+}$. This is confirmed by Fig. 9 .

\subsection{Outflows in NGC 6334 FIRII}

Straw et al. (1989b) found an arc of shocked vibrationally excited $\mathrm{H}_{2}$ enveloping the $\mathrm{H}$ II region NGC 6334-D (Sect. 4.1) that coincides with the molecular void. This was interpreted in terms of molecular gas swept away by an outflow powered by the central star(s). Brooks \& Whiteoak (2001) mention that so far no further observations have been made to confirm the presence of this outflow. Although we have not tested this idea by directly observing shocked $\mathrm{H}_{2}$ with high velocity resolution, we have collected well-resolved spectra from the $\mathrm{CO} J=1-0$ transition, which is the outflow tracer for cool molecular gas.

A detailed look at some CO spectra from the NGC 6334 FIR II region, presented in Fig. 10, reveals velocity components 


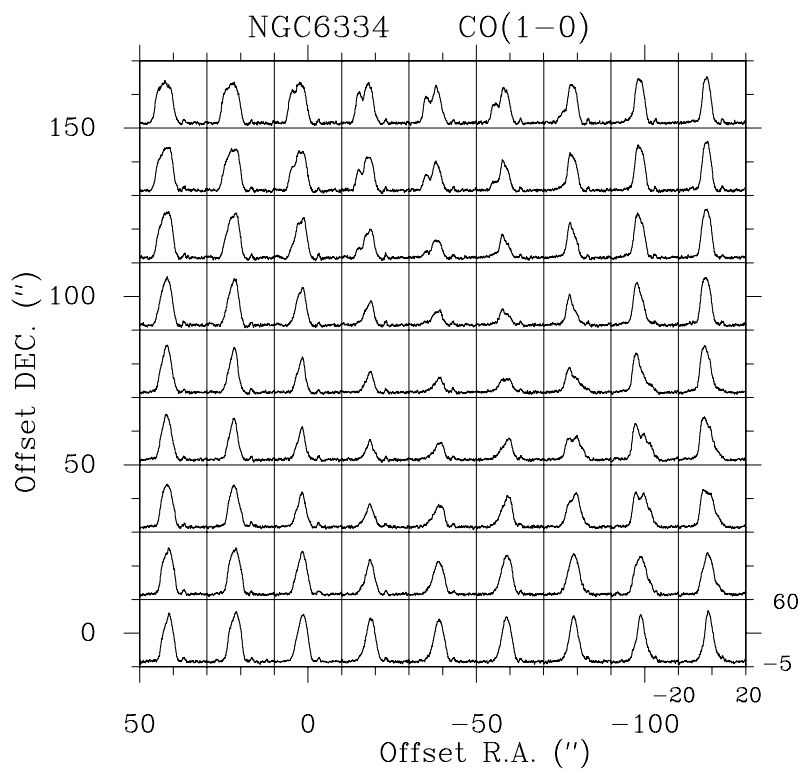

Fig. 10. CO $J=1-0$ spectra near the molecular void (for the full area, see Fig. A.1) showing red- and blueshifted emission in addition to the main component. The displayed velocity range is $-20 \mathrm{~km} \mathrm{~s}^{-1}<V_{\mathrm{LSR}}<$ $+20 \mathrm{~km} \mathrm{~s}^{-1}$. The $T_{\mathrm{mb}}$ range is $-5 \mathrm{~K}<T_{\mathrm{mb}}<+60 \mathrm{~K}$. The reference position is $\alpha_{1950}=17^{\mathrm{h}} 17^{\mathrm{m}} 30^{\mathrm{s}} .0, \delta_{1950}=-35^{\circ} 47^{\prime} 30^{\prime \prime}\left(\alpha_{2000}=17^{\mathrm{h}} 20^{\mathrm{m}} 51^{\mathrm{s}} .2\right.$, $\left.\delta_{2000}=-35^{\circ} 50^{\prime} 28^{\prime \prime}\right)$. The channel spacing is $0.11 \mathrm{~km} \mathrm{~s}^{-1}$.

in addition to the main feature discussed in Sect.3.1 and displayed in Fig. 2. Beside this main component at -6.5 to $-2 \mathrm{~km} \mathrm{~s}^{-1}$, there is a weak $+6 \mathrm{~km} \mathrm{~s}^{-1}$ feature that is only seen in $\mathrm{CO}$ and that is present in the N, E, S, and center. Apparently, this component is related neither to the molecular void nor to the ridge of dense molecular gas in the southwestern part of our map. In the north, there is a more notable component with a velocity of about $-10 \mathrm{~km} \mathrm{~s}^{-1}$. It is best seen in the ${ }^{13} \mathrm{CO}(2-1)$ line (Fig. A.1), but is also prominent in $\mathrm{CO}$ (Fig. 10), HCN, and $\mathrm{HCO}^{+}$, as well as in [CI] near the northern edge of the cross (Fig. 3). This component marks the southern tip of a kinematic feature extending southwards from NGC 6334 FIR I (see Fig. 11 of Kraemer \& Jackson 1999).

There also seems to be some evidence for a molecular outflow. Redshifted linewings of the "main" feature are apparent near the southwestern edge of the molecular void, not only in $\mathrm{CO}$ but also in ${ }^{13} \mathrm{CO}, \mathrm{HCN}$, and $\mathrm{HCO}^{+}$. Figure 11 shows the $\mathrm{CO}$ map of this redshifted emission. The redshifted linewing extends from the stellar sources IRS-23 and IRS-24 southwards, along the western edge of the molecular cloud ridge (see Fig. 1) that shows high (column) densities (Sect. 3.1). The corresponding blue-shifted linewings are, however, missing (Fig. 11). Furthermore, there is no indication of an outflow associated with the vibrationally excited $\mathrm{H}_{2}$ gas observed by Straw \& Hyland (1989b).

\section{7. ${ }^{18} \mathrm{O} /{ }^{17} \mathrm{O}$ ratios}

The determination of CNO isotope ratios plays a key role in our understanding of stellar nucleosynthesis and chemical evolution (e.g., Wilson \& Rood 1994). One of the most interesting CNO isotope ratios is that of the two rare oxygen nuclei, ${ }^{18} \mathrm{O}$ and ${ }^{17} \mathrm{O}$. With ${ }^{18} \mathrm{O}$ being mainly synthesized in massive stars $\left(\sim 8-20 M_{\odot}\right)$ and ${ }^{17} \mathrm{O}$ originating predominantly from lower mass stars (e.g., Hoffman et al. 2001; Stoesz \& Herwig 2003), their abundance ratio may become a tracer of material ejected by high-mass stars.

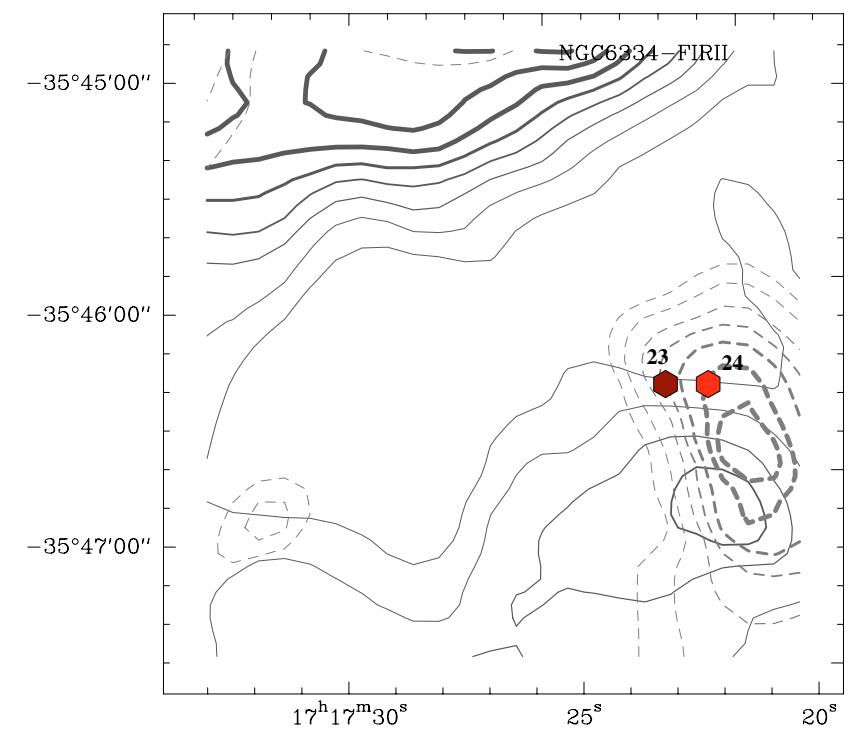

Fig. 11. Map of the red- and blueshifted $\mathrm{CO}$ components. Velocity ranges are $-14 \mathrm{~km} \mathrm{~s}^{-1}<V_{\mathrm{LSR}}<-8 \mathrm{~km} \mathrm{~s}^{-1}$ for the blue linewings (solid contours) and $2 \mathrm{~km} \mathrm{~s}^{-1}<V_{\mathrm{LSR}}<12 \mathrm{~km} \mathrm{~s}^{-1}$ for the red linewings (dashed contours). Contour levels are $30 \%-90 \%$ of the integrated peak emission of $51 \mathrm{~K} \mathrm{~km} \mathrm{~s}^{-1}$ (blue) and $41 \mathrm{~K} \mathrm{~km} \mathrm{~s}^{-1}$ (red) in steps of $10 \%$. The two hexagonal dots mark the stellar sources IRS23 and IRS24.

Observing the $J=1-0$ lines of $\mathrm{C}^{18} \mathrm{O}$ and $\mathrm{C}^{17} \mathrm{O}$ has the advantage that both lines tend to be optically thin and that measured ratios are small $(<10)$ so that required observational sensitivities are not extremely different. Fractionation due to ion exchange reactions (see Watson et al. 1976 for the stable carbon isotopes) does not play a major role (Langer et al. 1984). Hence the integrated intensity ratio is a good measure of ${ }^{18} \mathrm{O} /{ }^{17} \mathrm{O}$ ratios (see Wouterloot et al. 2005, for an extensive multi-level analysis). While the ratio is small $(1.6 \pm 0.3)$ in the Large Magellanic Cloud, in the nuclear starbursts of NGC 253 and NGC 4945 ${ }^{18} \mathrm{O} /{ }^{17} \mathrm{O}$ ratios of $\sim 6.5$ have been measured (Heikkilä et al. 1998; Harrison et al. 1999; Wang et al. 2004). These results indicate that the ratio does not only depend on the relative importance of massive versus less massive stars, but that metallicity also plays a role. In the Galaxy, the ratio is of order 4 (Table 4), but there is a clear discrepancy with the solar system (5.5; e.g., Anders $\&$ Grevesse 1989) that may have been formed out of material enriched by nearby massive stars (e.g., Lyons \& Young 2005).

From our spectral data integrated intensities were obtained by Gaussian fitting or, in cases of poor signal-to-noise ratios, by the first moment (area). In the latter cases we determined integrated intensities by summing line intensities over the velocity interval -13 to $+3 \mathrm{~km} \mathrm{~s}^{-1} \cdot \mathrm{C}^{18} \mathrm{O} / \mathrm{C}^{17} \mathrm{O}$ ratios were calculated for those 152 of the 169 positions that show $\mathrm{C}^{17} \mathrm{O}$ signal-to-noise ratios $\gtrsim 3$.

In Fig. 12, the ratios are plotted against the corresponding integrated intensities of $\mathrm{C}^{17} \mathrm{O}$. The ratios show significant scatter at low integrated intensities due to noise in the $\mathrm{C}^{17} \mathrm{O}$ spectra. For clouds filling the beam, total $\mathrm{C}^{18} \mathrm{O}$ and $\mathrm{C}^{17} \mathrm{O}$ column densities are proportional to $v^{-2}$ times the integrated line intensity (e.g., Linke et al. 1977). Measured line intensity ratios thus have to be corrected: $\mathrm{C}^{18} \mathrm{O} / \mathrm{C}^{17} \mathrm{O}($ corrected $)=\left(v_{\mathrm{C} 17 \mathrm{O}} / v_{\mathrm{C} 18 \mathrm{O}}\right)^{2} \times$ $\mathrm{C}^{18} \mathrm{O} / \mathrm{C}^{17} \mathrm{O}$ (observed). The correction factor is 1.047. Applying this factor, we obtain abundance ratios of $4.32 \pm 0.13$ averaging all plotted data points, $4.13 \pm 0.13$ for the positions with $I\left(\mathrm{C}^{17} \mathrm{O}\right)>1 \mathrm{~K} \mathrm{~km} \mathrm{~s}^{-1}, 4.02 \pm 0.10$ for those with $I\left(\mathrm{C}^{17} \mathrm{O}\right)>$ $2 \mathrm{~K} \mathrm{~km} \mathrm{~s}^{-1}$, and $4.13 \pm 0.12$ for those with $I\left(\mathrm{C}^{17} \mathrm{O}\right)>3 \mathrm{~K} \mathrm{~km} \mathrm{~s}^{-1}$, 
Table 4. Measured ${ }^{18} \mathrm{O} /{ }^{17} \mathrm{O}$ ratios ${ }^{a}$.

\begin{tabular}{cccc}
\hline \hline Source & Ratio & Notes & Reference \\
\hline Galactic disk & $3.65 \pm 0.15$ & frequency corrected value & Penzias (1981) \\
Nearby dark clouds & $3.2 \pm 0.2$ & frequency corrected value & Wilson et al. (1981) \\
Ophiuchus cloud complex (1 position) & $4.15 \pm 0.52$ & frequency corrected value & Bensch et al. (2001) \\
Taurus molecular cloud (648 positions) & $4.0 \pm 0.5$ & from a "simple model" & Ladd (2004) \\
Ophiuchus cloud complex (20 positions) & $3.53 \pm 0.11$ & frequency corrected value & Wouterloot et al. (2005) \\
Ophiuchus cloud complex (17 positions) & $4.07 \pm 0.32$ & assuming LTE & Wouterloot et al. (2005) \\
Ophiuchus cloud complex (21 positions) & $4.10 \pm 0.14$ & from an LVG model & Wouterloot et al. (2005) \\
NGC 6334 FIR II (169 positions) & $4.13 \pm 0.13$ & frequency corrected value & this paper \\
\hline
\end{tabular}

${ }^{a}$ Results are mainly derived from $\mathrm{C}^{18} \mathrm{O} / \mathrm{C}^{17} \mathrm{O} J=1-0$ integrated intensity ratios. Exceptions are Bensch et al. (2001), who measured a ${ }^{13} \mathrm{C}^{18} \mathrm{O} /{ }^{13} \mathrm{C}^{17} \mathrm{O} J=1-0$ ratio, and Wouterloot et al. (2005), who analyzed the $J=1-0,2-1$, and $3-2$ lines of $\mathrm{C}^{18} \mathrm{O}$ and $\mathrm{C}^{17} \mathrm{O}$ including some lines-of-sight with moderately optically thick $\mathrm{C}^{18} \mathrm{O}$ emission.

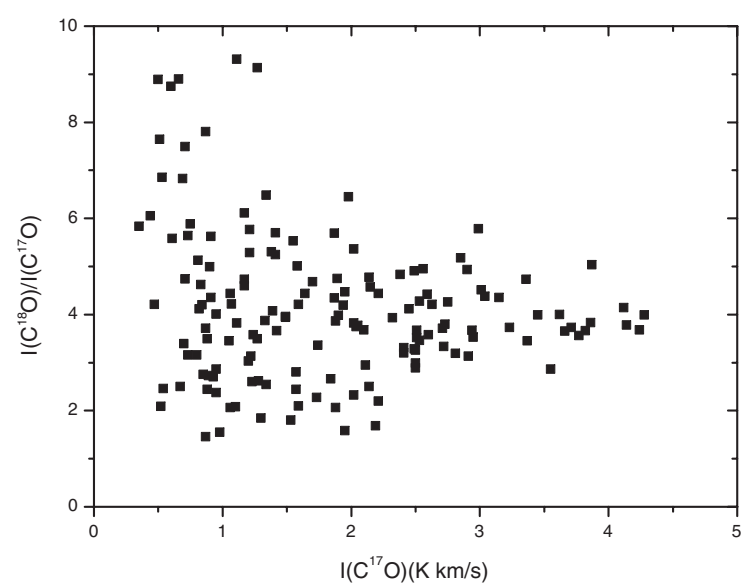

Fig. 12. $\mathrm{C}^{18} \mathrm{O} / \mathrm{C}^{17} \mathrm{O} J=1-0$ integrated line intensity ratios versus $\mathrm{C}^{17} \mathrm{O} J=1-0$ integrated intensity.

the errors denoting the standard deviation of the mean. While the number of values obtained toward positions with increasing minimum intensity is decreasing, the scatter in the individual ratios is also decreasing because of higher signal-to-noise ratios. Thus standard deviations remain almost the same.

The derived values are in excellent agreement with those of the LTE and LVG (large velocity gradient) model results presented by Wouterloot et al. (2005) from the Ophiuchus cloud, with that reported by Bensch et al. (2001) from a ${ }^{13} \mathrm{C}^{18} \mathrm{O} /{ }^{13} \mathrm{C}^{17} \mathrm{O}$ intensity ratio toward the same cloud complex (see Table 4), and with those of Ladd et al. (2004) from the similarly nearby Taurus molecular cloud. Our frequency-corrected values are higher than those of the "only" frequency-corrected values of Wouterloot et al. (2005), but this is not a serious disagreement. Wouterloot et al. (2005) purposely observed positions with a wide range of column densities, including some positions where the $\mathrm{C}^{18} \mathrm{O}$ opacity was not negligible. In these cases, the linear correlation between line strength and column density breaks down and abundance ratios become larger than frequency-corrected line intensity ratios. This effect may also play a role in the data presented by Penzias (1981) and Wilson et al. (1981).

Our data do not hint at optical depth effects. The $\mathrm{C}^{18} \mathrm{O} / \mathrm{C}^{17} \mathrm{O}$ ratio stays constant up to the positions with highest line intensity. ${ }^{18} \mathrm{O}$ enriched material from massive stars and isotope selective photodissociation would increase the measured line intensity ratios. High opacities in $\mathrm{C}^{18} \mathrm{O}$ would reduce them. Only having measured the $J=1-0$ lines of $\mathrm{C}^{18} \mathrm{O}$ and $\mathrm{C}^{17} \mathrm{O}$, we cannot firmly exclude that these three effects precisely cancel each other. However, in view of our estimate of $\tau\left({ }^{13} \mathrm{CO} J=\right.$ $1-0) \sim 0.3$ in Sect. 4.4 and following the principle of Occam's razor, such a scenario is farfetched. Line saturation and isotope selective photodissociation can thus be neglected.

The aim of the isotope ratio measurements was to find out, whether ${ }^{18} \mathrm{O}$ enrichment can be seen at the very edge of the molecular void. This is neither apparent in Fig. 12 nor is there any such tendency when only analyzing those positions that are located next to the $\mathrm{H}$ II region. At the eastern edge of the void, averaging spectra at positions with offsets $(0,40),(0,60)$, $(0,80)$, and $(0,100)$ (see Figs. 11 and A.1), we find a frequencycorrected ratio of $3.0 \pm 0.2$. Toward its western edge, at positions $(-80,40),(-80,60),(-80,80),(-80,100)$, and $(-80,120)$, we find $2.3 \pm 0.1$. The latter ratio appears to be too low because toward one position, $\mathrm{C}^{17} \mathrm{O}$ but not $\mathrm{C}^{18} \mathrm{O}$, is detected. Thus pointing uncertainties might play a role. The ring with radius $40^{\prime \prime}-50^{\prime \prime}$ and density $n\left(\mathrm{H}_{2}\right) \sim 10^{3} \mathrm{~cm}^{-3}$ around the center of the void (representing the inner edge of the cloud, see Sects. 3 and 4.4) contains about $5-10 M_{\odot}$, which is similar to the mass formerly filling the ionized bubble, if we assume the same average density. Depending on stellar mass and evolutionary stage, the gas at the edge of the void might therefore be significantly affected by the ejecta (possibly also amounting to several $M_{\odot}$ ) of young massive stars. We note, however, that the ${ }^{18} \mathrm{O} /{ }^{17} \mathrm{O}$ ratios near the molecular void suggest the presence of lower, not higher ${ }^{18} \mathrm{O} /{ }^{17} \mathrm{O}$ ratios when comparing them with values from interstellar clouds in the solar neighborhood. This does not agree with the high values known to characterize extragalactic nuclear starbursts.

\subsection{Formation and evolutionary stage of the molecular void}

As already indicated, in nuclear starbursts of nearby galaxies ${ }^{18} \mathrm{O}$ is enriched with respect to ${ }^{17} \mathrm{O}$ (Harrison et al. 1999; Wang et al. 2004). In the Orion OB association the youngest subassociations show higher oxygen abundances than the older subassociations (Cunha \& Lambert 1992). The solar system shows a significantly enhanced ${ }^{18} \mathrm{O} /{ }^{17} \mathrm{O}$ ratio relative to interstellar values, presumably because of ${ }^{18} \mathrm{O}$ enrichment just prior to its formation (Henkel \& Mauersberger 1993). Why do we not measure enhanced ${ }^{18} \mathrm{O} /{ }^{17} \mathrm{O}$ ratios near the edge of the NGC 6334-FIRII molecular cloud? Following Heger \& Langer (2000), Hoffman et al. (2001), and Rauscher et al. (2002), stars with zero age main sequence masses up to $20 M_{\odot}$ show high ${ }^{18} \mathrm{O}$ yields during core collapse. With a highly obscured O6.5 star (IRS-24; $M \sim 40 M_{\odot}$ ) likely being responsible for the molecular void (Sect. 4.1), stars with $\$ 20 M_{\odot}$ had, however, not yet sufficient time to reach the supernova stage of evolution. For rotating 
massive stars, Heger \& Langer (2000) predict enhanced ${ }^{17} \mathrm{O}$ and depleted ${ }^{18} \mathrm{O}$ surface abundances during the pre-supernova stages, at the time of the first dredge up and even before, at core hydrogen exhaustion. Prior to core collapse, a low ${ }^{18} \mathrm{O} /{ }^{17} \mathrm{O}$ ratio in the affected interstellar medium is therefore consistent with theory.

The lifetime of an O6.5 star is $\sim 4.5 \times 10^{6} \mathrm{yr}$ (e.g., Massey 2005), providing an upper age limit for the molecular void. There are also indications that the region is not extremely young (Sect. 4.1). The extent of the FIR emission and the size of the radio continuum source $(\lambda 16 \mathrm{~cm}$ diameter: $\sim 1 \mathrm{pc}$; e.g., Brooks $\&$ Whiteoak 2001) are large when compared with the other main sources of activity in NGC 6334. Remarkable is the regular structure of the radio continuum shell. Apparently, while being very clumpy on small scales (Sect. 4.5), the surrounding parental molecular cloud must have been quite homogeneous on larger scales. There is no hint for the presence of a "champagne phase" expected when the ionized shell expands to the edge of the cloud.

As already indicated in Sect. 1, H II regions expanding into an approximately homogeneous medium should show, at later stages of their evolution, a decelerated subsonic "D-type" ionization front that is slowly following a shock front farther out. This is likely what we are viewing. While thermal continuum radiation encompasses an almost circular area of diameter $2^{\prime}$, even further to the east there is the prominent arc of warm presumably shocked $\mathrm{H}_{2}$ gas. Since $\mathrm{CO}$ is not providing any evidence for outflowing material, $\mathrm{H}_{2}$ may not trace the location of an outflow (as suggested by Straw \& Hyland 1989b), but instead marks the shockfront compressing neutral gas beyond the periphery of the evolved H II region surrounding IRS-24.

In Sects. 4.4 and 4.5 we obtained cloud densities of $10^{3} \mathrm{~cm}^{-3}$ from $\mathrm{CO}$ and $\gtrsim 10^{5} \mathrm{~cm}^{-3}$ from $\mathrm{HCN}$. For an intermediate value, $n\left(\mathrm{H}_{2}\right)=10^{4} \mathrm{~cm}^{-3}$, Fig. $4 \mathrm{c}$ provides the size of the "initial" Strömgren sphere, $0.35 \mathrm{pc}$ or $0 \mathbf{\prime}^{\prime} 7$. This is smaller than the observed sizes of the molecular void and $\mathrm{H}$ II region, $\sim 1.5$ and $2^{\prime}$ (Figs. 1 and 4). Since an HII region can expand up to about five times its Strömgren radius (until pressure equilibrium is attained) if the ionizing star does not move off the main sequence before (e.g., Yorke 1986), there is no serious contradiction between models and observations. In case the ionized gas just occupies the "initial" Strömgren sphere, we obtain an average density of $n\left(\mathrm{H}_{2}\right) \sim 2 \times 10^{3} \mathrm{~cm}^{-3}$ (e.g., Tielens 2005, p. 231). This is consistent with our density estimate from $\mathrm{CO}$, which represents the bulk of the molecular gas surrounding the void.

In between the outer shell of shock heated vibrationally excited $\mathrm{H}_{2}$ (Straw \& Hyland 1989b) and the ionized region further inside, conditions should be optimal for PDRs. While $[\mathrm{CI}]$ is weak in the molecular void that is readily observed in the radio continuum through thermal free-free emission, [C II] is quite prominent (Kraemer et al. 2000). Suitable discriminators between PDRs and shocks are the absolute and relative intensities of the [C II] $158 \mu \mathrm{m}$ and [O I] $63 \mu \mathrm{m}$ fine structure lines. One can find weak [C II] and strong [O I] emission $(I([\mathrm{OI}]) / I([\mathrm{C} \mathrm{II}]) \gg 10)$ in shocks, while the reverse holds for PDRs (Genzel 1991). Observations of far-infrared fine-structure lines in NGC 6334, [C II] $158 \mu \mathrm{m}$, and [O I] $63 \mu \mathrm{m}$ and $145 \mu \mathrm{m}$, strongly support the presence of PDRs that must be widespread. The most intense [C II] emission in NGC 6334 is associated with the FIR II region (Boreiko \& Betz 1995; Kraemer et al. 1998, 2000). $I([\mathrm{O} \mathrm{I}]) / I([\mathrm{C} \mathrm{II}]) \sim 3$ (Kraemer et al. 1998).

\section{Summary}

Having performed observations of molecular and atomic lines in the galactic star-forming region NGC 6334, we find the following main results:

1. The large-scale distribution of ${ }^{13} \mathrm{CO} J=1-0$ emission over the entire star forming-region shows strong peaks toward FIR I, IV, and V, while FIR II and III are merely associated with ridge emission extending southwards from FIR I and northeastwards from FIR IV, respectively.

2. A detailed study of the eastern part of the most luminous source of far-infrared emission, NGC 6334 FIR II, reveals a molecular void associated with the $\mathrm{HII}$ region NGC 6334-D. Molecular and [C I] excitation indicate $T_{\text {kin }} \sim$ $40-50 \mathrm{~K}$ in the surrounding molecular cloud, while toward the void itself $T_{\text {kin }} \gtrsim 200 \mathrm{~K}$. Molecular column densities and measured obscuration suggest that the exciting star IRS-24 (presumably of type O6.5) is located behind the bulk of the molecular column.

3. The CO spectra show redshifted velocity wings south of IRS-24, but the corresponding blueshifted feature is missing.

4. ${ }^{18} \mathrm{O} /{ }^{17} \mathrm{O}$ abundance ratios reflecting the ${ }^{18} \mathrm{O} /{ }^{17} \mathrm{O}$ isotope ratio were obtained from measurements of the $J=1-0$ lines of $\mathrm{C}^{18} \mathrm{O}$ and $\mathrm{C}^{17} \mathrm{O}$. The mean ratio is $4.13 \pm 0.13$, in good agreement with recent measurements toward nearby molecular clouds. At the edge of the molecular void, however, ratios might be smaller.

5. The regular morphology of the ionized gas of the H II region indicates the presence of a rather homogeneous parental molecular cloud on parsec scales. The D-type ionization front (from observations of the radio continuum) and presumably also the shock front farther out in the molecular gas (from measurements of vibrationally excited $\mathrm{H}_{2}$ ) can be identified. The age of the $\mathrm{H}$ II region must be $<5 \times 10^{6} \mathrm{yr}$.

6. The $[\mathrm{CI}]$ distribution follows that of the molecular gas and does not show a bright rim at the inner portion of the molecular shell. This may be interpreted in terms of a high degree of small-scale clumping, with PDRs reaching deep into the dense surrounding cloud. Such small scale clumping is indicated by the different densities obtained from $\mathrm{CO}$ and $\mathrm{HCN}$ data $\left(n\left(\mathrm{H}_{2}\right) \sim 10^{3}\right.$ and $\gtrsim 10^{5} \mathrm{~cm}^{-3}$, respectively). Observationally, however, the [C I] lines do not provide any direct hint for a close connection with PDRs.

So far, the velocity field of the shocked $\mathrm{H}_{2}$ gas has not been measured. Additional observations of tracers of shocked molecular gas like $\mathrm{SiO}$ (Gueth et al. 1998) or HNCO (Zinchenko et al. 2000) would also be desirable. A dedicated experiment, investigating the potential anomaly in the ${ }^{18} \mathrm{O} /{ }^{17} \mathrm{O}$ ratio near the edge of the void to further constrain enrichment by ejecta from massive stars would be another worthwhile project.

Acknowledgements. We wish to thank the anonymous referee for useful comments. J. S. Zhang acknowledges support by the exchange program between the Chinese Academy of Sciences and the Max-Planck-Gesellschaft.

\section{References}

Anders, E., \& Grevesse, N. 1989, Geochim. Cosmochim. Acta, 53, 197 Bensch, F., Pak, I., Wouterloot, J. G. A., Klapper, G., \& Winnewisser, G. 2001, ApJ, 562, L185

Bohlin, R. C., Savage, B. D., \& Drake, J. F. 1978, ApJ, 224, 132

Booth, R. S., Delgado, G., Hagström, M., et al. 1989, A\&A, 216, 315

Boreiko, R. T., \& Betz, A. L. 1995, ApJ, 454, 307 
Brooks, K. J., \& Whiteoak, J. B. 2001, MNRAS, 320, 465

Brouillet, N., Muller, S., Herpin, F., Braine, J., \& Jacq, T. 2005, A\&A, 429, 153

Burton, M. G., Ashley, R. D., Marks, R. D., et al. 2000, ApJ, 542, 359

Bykov, A. M., Krassilchtchikov, A. M., Uvarov, Y. A., et al. 2006, A\&A, 449, 917

Caproni, A., Abraham, Z., \& Vilas-Boas, J. W. S. 2000, A\&A, 361, 685

Carral, P., Kurtz, S. E., Rodríguez, L. F., et al. 2002, ApJ, 123, 2574

Chin, Y.-N., Henkel, C., Whiteoak, J. B., et al. 1997, A\&A, 317, 548

Churchwell, E. 2002, ARA\&A, 40, 27

Christopher, M. H., Scoville, N. Z., Stolovy, S. R., \& Yun, M. S. 2005, ApJ, 622, 346

Cunha, K., \& Lambert, D. L. 1992, ApJ, 399, 586

Downes, D. 1989, in Introductory Course in galaxies' evolution and observational astronomy, ed. I. Appenzeller, H. Habing, \& P. Lena (Heidelberg: Springer-Verlag), 353

Ezoe, Y., Kokubin, M., Makishima, K., Sekimoto, Y., \& Matsuzaki, K. 2006, ApJ, 638, 860

Flower, D. R. 2001, J. Phys. B: At. Mol. Opt. Phys., 34, 1

Franco, J., Tenorio-Tagle, G., \& Bodenheimer, P. 1990, ApJ, 349, 126

Fuente, A., Martín-Pintado, J., Cernicharo, J., \& Bachiller, R. 1993, A\&A, 276 473

Gezari, D. 1982, ApJ, 259, L29

Gezari, D., \& Blitz, L. 1990, in Submillimetre Astronomy, ed. G. D. Watt, \& A. S. Webster (Kluwer), 173

Genzel, R. 1991, in The physics of star formation and early stellar evolution, ed C. J. Lada, \& N. D. Kylafis, 155

Gueth, F., Guilloteau, S., \& Bachiller, R. 1998, A\&A, 333, 287

Guilloteau, S., \& Lucas, R. 2000, in Imaging at Radio through Submillimeter Wavelengths, ASP Conf. Ser., 217, 299

Güsten, R., Nyman, L. Å., Schilke, P., et al. 2006, A\&A, 454, L13

Harrison, A., Henkel, C., \& Russell, A. 1999, MNRAS, 303, 157

Heger, A., \& Langer, N. 2000, ApJ, 544, 1016

Heikkilä, A., Johansson, L. E. B., \& Olofsson, H. 1998, A\&A, 332, 493

Helfer, T. T., \& Blitz, L. 1997, ApJ, 478, 233

Henkel, C., \& Mauersberger, R. 1993, A\&A, 274, 730

Heyminck, S., Kasemann, C., Güsten, R., de Lange, G., \& Graf, U. U. 2006, A\&A, 454, L21

Hoffman, R. D., Woosley, S. E., \& Weaver, T. A. 2001, ApJ, 549, 1085

Hollenbach, D. J., \& Tielens, A. G. G. M. 1997, ARA\&A. 35, 179

Hunter, T. R., Brogan, C. L., Megeath, S. T., Menten, K. M., \& Beuther, S. 2006, ApJ, 649, 888

Ikeda, M., Oka, T., Tatematsu, K., Sekimoto, Y., \& Yamamoto, S. 2002, ApJS, 139,467

Klein, B., Philipp, S. D., Krämer, I., et al. 2006, A\&A, 454, L29

Kraemer, K. E., \& Jackson, J. M. 1999, ApJS, 124, 439

Kraemer, K. E., Jackson, J. M., \& Lane, A. P. 1998, ApJ, 503, 785

Kraemer, K. E., Jackson, J. M., Lane, A. P., \& Paglione, T. A. D. 2000, ApJ, 542, 946

Kuiper, T. B. H., Peters III, W. L., Foster, J. R., Gardner, F. F., \& Whiteoak, J. B. 1995, ApJ, 446, 692

Ladd, E. F. 2004, ApJ, 610, 320
Langer, W. D., Graedel, T. E., Frerking, M. A., \& Armentrout, P. B. 1984, ApJ, 277,581

Leurini, S., Schilke, P., Parise, F., et al. 2006, A\&A, 454, L83

Linke, R. A., Goldsmith, P. F., Wannier, P. G., Wilson, R. W., \& Penzias, A. A. 1977, ApJ, 214, 50

Loughran, L., McBreen, B., Fazio, G. G., et al. 1986, ApJ, 303, 629

Lyons, J. R., \& Young, E. D. 2005, Nature, 435, 317

Mao, R. Q., Henkel, C., Schulz, A., et al. 2000, A\&A, 358, 433

Massey, P. 2005, ARA\&A, 41, 15

McBreen, B., Fazio, G. G., Stier, M., \& Wright, E. L. 1979, ApJ, 232, L183

McCutcheon, W. H., Sandell, G., Matthews, H. E., et al. 2000, MNRAS, 316, 152

Meaburn, J., \& White, N. J. 1982, ApJ, 255, L55

Moran, J. M., \& Rodríguez, L. F. 1980, ApJ, 236, L159

Neckel, T. 1978, A\&A, 69, 51

Penzias, A. A. 1981, ApJ, 249, 518

Persi, P., Tapia, M., \& Roth, M. 2000, A\&A, 357, 1020

Pirogov, L. 1999, A\&A, 348, 600

Plume, R., Jaffe, D. T., Tatematsu, K., Evans II, N. J., \& Keene, J. 1999, ApJ, 512,768

Rauscher, T., Heger, A., Hoffman, R. D., \& Woosley, S. E. 2002, ApJ, 576, 323 Rodríguez, L., Cantó, J., \& Moran, J. 1982, ApJ, 255, 103

Rohlfs, K., \& Wilson, T. L. 1996, Tools of Radio Astronomy, 2nd Ed. (Berlin: Springer), 194

Sandell, G. 2000, A\&A, 358, 242

Sarma, A. P., Troland, T. H., Roberts, D. A., \& Crutcher, R. M. 2000, ApJ, 533, 271

Schilke, P., Comito, C., Thorwirth, S., et al. 2006, A\&A, 454, L41

Schneider, N., Simon, R., Kramer, C., et al. 2003, A\&A, 406, 915

Schöier, F. L., van der Tak, F. F. S., van Dishoeck, E. F., \& Black, J. H. 2005, A\&A, 432, 369

SEST Handbook 1993, European Southern Observatory Operating Manual No. 19, Version 1.0

Stoesz, J. A., \& Herwig, F. 2003, MNRAS, 340, 763

Straw, S. M., \& Hyland, A. R. 1989a, ApJ, 340, 318

Straw, S. M., \& Hyland, A. R. 1989b, ApJ, 342, 876

Straw, S. M., Hyland, A. R., \& McGregor, P. J. 1989, ApJS, 69, 99

Stutzki, J., Graf, U. U., Haas, S., et al. 1997, ApJ, 477, L33

Tenorio-Tagle, G. 1988, ARA\&A, 26, 145

Tielens, A. G. G. M. 2005, The physics and chemistry of the interstellar medium (Cambridge: Cambridge University Press)

Wang, M., Henkel, C., Chin, Y.-N., et al. 2004, A\&A, 422, 883

Watson, W. D., Anicich, V. G., \& Huntress, W. T. 1976, ApJ, 205, L165

Weiß, A., Henkel, C., Downes, D., \& Walter, F. 2003, A\&A, 409, L41

Wilson, R. W., Langer, W. D., \& Goldsmith, P. F. 1981, ApJ, 243, L47

Wilson, T. L., \& Rood, R. T. 1994, ARA\&A, 32, 191

Wilson, T. L., \& Walmsley, C. M. 1989, A\&AR, 1, 141

Wouterloot, J. G. A., Brand, J., \& Henkel, C. 2005, A\&A, 430, 549

Yorke, H. W. 1986, ARA\&A, 24, 49

Zinchenko, I., Henkel, C., \& Mao, R. Q. 2000, A\&A, 361, 1079 


\section{Online Material}




\section{Appendix A: Observed SEST spectra}
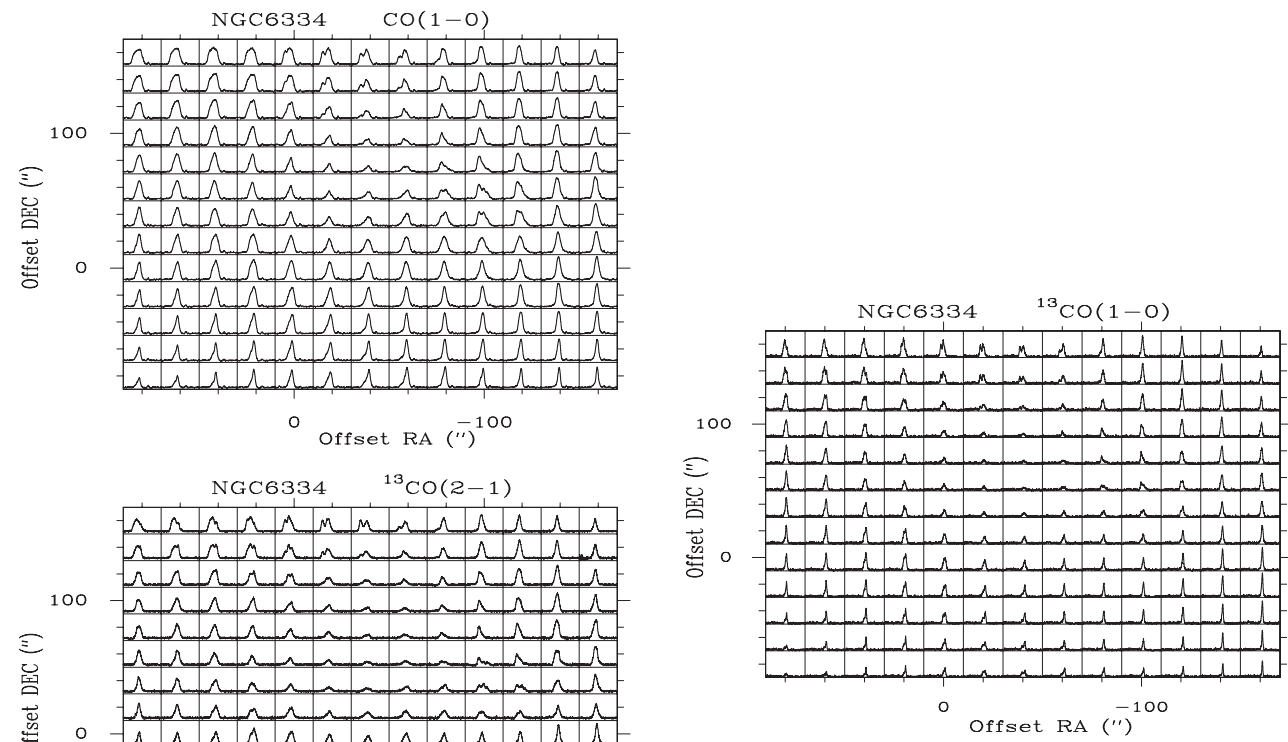

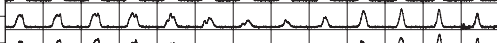

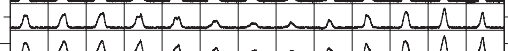

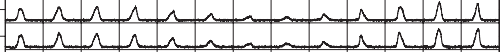
$1 \mathrm{n}$ r n

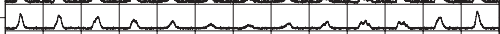
rerararanerararar

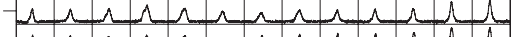

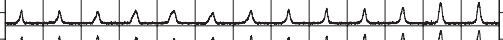

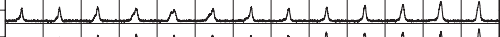

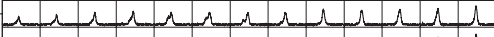

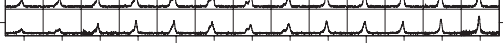
O Offset RA (") ${ }^{100}$ NGC6334 $\quad \mathrm{C}^{17} \mathrm{O}(1-\mathrm{O})$
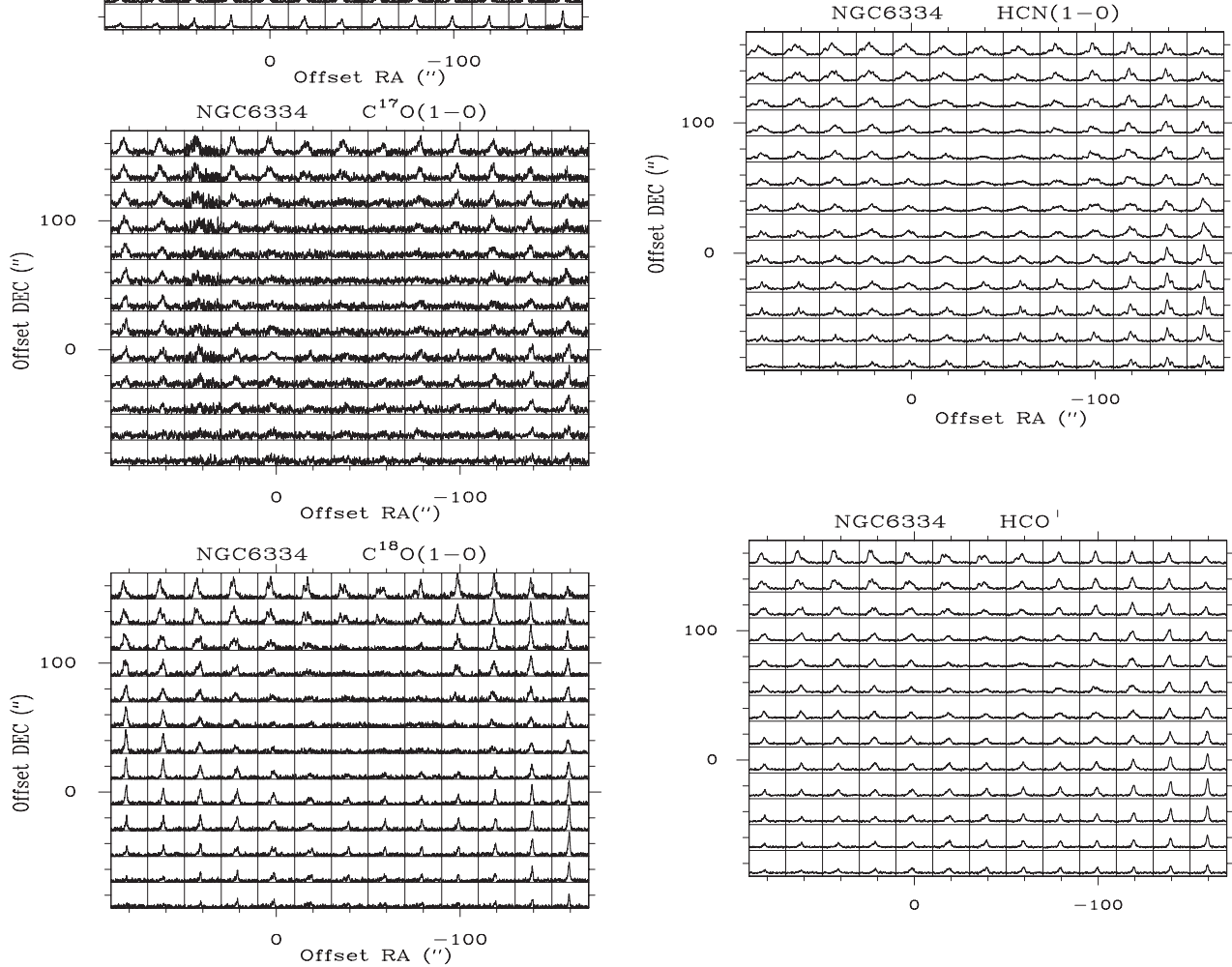

$\mathrm{NGC6334} \mathrm{HCO}^{\prime}$

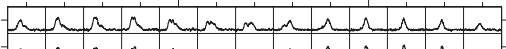

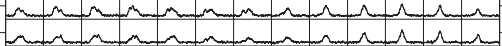

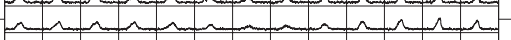

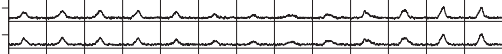

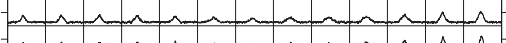

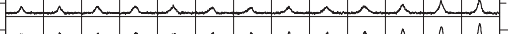
-

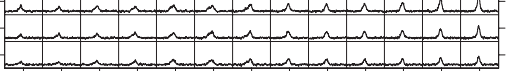
o $-100$

Fig. A.1. Spectra of seven molecular transitions across the NGC 6334 FIR II region. Left (from top to bottom): ${ }^{12} \mathrm{CO}(1-0),{ }^{13} \mathrm{CO}(2-1)$, $\mathrm{C}^{17} \mathrm{O}$, and $\mathrm{C}^{18} \mathrm{O}$; right: ${ }^{13} \mathrm{CO}(1-0), \mathrm{HCN}(1-0)$, and $\mathrm{HCO}^{+}(1-0)$. The $(0,0)$ position of the maps is $\alpha_{1950}=17^{\mathrm{h}} 17^{\mathrm{m}} 30^{\mathrm{s}} .0$ and $\delta_{1950}=-35^{\circ} 47^{\prime} 30^{\prime \prime}$ $\left(\alpha_{2000}=17^{\mathrm{h}} 20^{\mathrm{m}} 51^{\mathrm{s}} .2\right.$ and $\left.\delta_{2000}=-35^{\circ} 50^{\prime} 28^{\prime \prime}\right)$. The $x$-axis of each individual spectrum denotes the local standard of rest velocity from -20 to $+20 \mathrm{~km} \mathrm{~s}^{-1}$. Main beam brightness temperature scales are -5 to $60 \mathrm{~K}(\mathrm{CO}),-1$ to $25 \mathrm{~K}\left({ }^{13} \mathrm{CO} J=1-0\right),-5$ to $45 \mathrm{~K}\left({ }^{13} \mathrm{CO} J=2-1\right),-0.2$ to $1 \mathrm{~K}$ $\left(\mathrm{C}^{17} \mathrm{O}\right),-0.2$ to $4 \mathrm{~K}\left(\mathrm{C}^{18} \mathrm{O}\right)$, and -2 to $13 \mathrm{~K}\left(\mathrm{HCN}\right.$ and $\left.\mathrm{HCO}^{+}\right)$. 